\title{
Probing top quark neutral couplings in the Standard Model Effective Field Theory at NLO in QCD
}

\author{
Olga Bessidskaia Bylund, ${ }^{a}$ Fabio Maltoni, ${ }^{b}$ loannis Tsinikos, ${ }^{b}$ Eleni Vryonidou $^{b}$ and \\ Cen Zhang ${ }^{c}$ \\ a Oskar Klein Centre and Department of Physics, Stockholm University, \\ SE-10691 Stockholm, Sweden \\ ${ }^{b}$ Centre for Cosmology, Particle Physics and Phenomenology (CP3), \\ Université catholique de Louvain, \\ B-1348 Louvain-la-Neuve, Belgium \\ ${ }^{c}$ Department of Physics, Brookhaven National Laboratory, \\ Upton, NY 11973, U.S.A. \\ E-mail: olga.bylund@cern.ch, fabio.maltoni@uclouvain.be, \\ ioannis.tsinikos@uclouvain.be, eleni.vryonidou@uclouvain.be, \\ cenzhang@bnl.gov
}

ABSTRACT: Top quark pair production in association with a $Z$-boson or a photon at the LHC directly probes neutral top-quark couplings. We present predictions for these two processes in the Standard Model (SM) Effective Field Theory (EFT) at next-to-leading order (NLO) in QCD. We include the full set of CP-even dimension-six operators that enter the top-quark interactions with the SM gauge bosons. For comparison, we also present predictions in the SMEFT for top loop-induced $H Z$ production at the LHC and for $t \bar{t}$ production at the ILC at NLO in QCD. Results for total cross sections and differential distributions are obtained and uncertainties coming from missing higher orders in the strong coupling and in the EFT expansions are discussed. NLO results matched to the parton shower are available, allowing for event generation to be directly employed in an experimental analyses. Our framework provides a solid basis for the interpretation of current and future measurements in the SMEFT, with improved accuracy and precision.

Keywords: Beyond Standard Model, Effective field theories

ARXIV EPRINT: 1601.08193 


\section{Contents}

1 Introduction 1

2 Effective operators $\quad 3$

3 Calculation setup $\quad 5$

4 Results for $t \bar{t} Z, t \bar{t} \gamma$ and $t \bar{t} \mu^{+} \mu^{-} \quad 6$

4.1 Inclusive $t \bar{t} Z, t \bar{t} \gamma$ and $t \bar{t} \mu^{+} \mu^{-}$results $\quad 6$

$\begin{array}{lll}4.2 & \text { Differential distributions } & 13\end{array}$

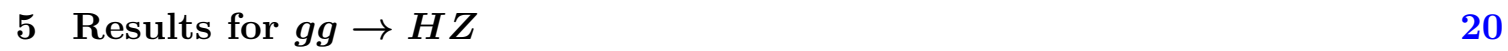

6 Results for the ILC $\quad 24$

$\begin{array}{lll}7 & \text { Theoretical uncertainties } & 25\end{array}$

$\begin{array}{lll}8 & \text { Discussion } & 27\end{array}$

$9 \quad$ Summary and conclusions $\quad 32$

A Connection with "anomalous coupling" approach 33

B Ratios for comparing with measurements $\quad 34$

B.1 ATLAS $-t \bar{t} Z \quad 34$

B.2 CMS $-t \bar{t} \gamma \quad 34$

\section{Introduction}

Top quark measurements are an important priority in Run II at the LHC. Results from the Tevatron and the first run of the LHC at 7 and $8 \mathrm{TeV}$ have yielded precise measurements of the main top quark production channels, i.e. top-anti-top production and single top production. At the LHC, the high energy and luminosity open up new possibilities to access rarer production processes, such as the associated production of top pairs with a vector boson. These processes are particularly interesting, as they provide the first probe of the neutral couplings of the top quark to the electroweak gauge bosons, which were not accessible at the Tevatron due to their high production thresholds. Therefore these channels could give important information about the top quark, which are complementary to top-pair and single-top production measurements as well as the top decay measurements. Measurements of $t \bar{t} \gamma$ have been performed at the Tevatron by CDF [1], and at the LHC by CMS [2] and by ATLAS [3]. Results for $t \bar{t} Z$ and $t \bar{t} W$ by CMS appear in $[4,5]$ and by ATLAS in [6]. 
Measurements of these processes allow us to search for deviations from the Standard Model (SM) predictions. While these deviations are often interpreted in terms of anomalous top couplings, the SM Effective Field Theory (SMEFT) provides a much more powerful framework [7-9]. In this approach possible deviations can be consistently and systematically described by the effects of higher-dimensional operators of the SM fields. By employing global analyses [10-12], experimental results can be used to determine the size of the deviations due to each effective operator. The established deviations can then be consistently evolved up to high scales, and matched to possible new physics scenarios. In the absence of convincing evidence for new resonance states, the EFT provides the most model-independent approach to a global interpretation of measurements.

With Run-II of the LHC, more and more precise measurements in the top-quark sector can be expected. In this respect, theoretical predictions matching the foreseeable precision of the experimental determinations are required to extract correct and useful information about deviations in the top-quark sector. For this reason, recently fully differential NLO QCD corrections to top-quark processes within the top quark EFT have started to become available, for example for the top-decay processes including the main decay channel and the flavor-changing channels $[13,14]$, and for single-top production triggered by flavor-changing neutral interactions of the top [15]. More recently, the two main production channels in the SM, top-quark pair production and single top production, have also become available at dimension-six at NLO in QCD $[16,17]$. QCD corrections are found to have nontrivial impact on SMEFT analyses [17].

In this work, we pursue this line of research further. We provide NLO QCD predictions for the $t \bar{t} Z$ and $t \bar{t} \gamma$ channels at the LHC and $t \bar{t}$ production at the ILC, including the full set of dimension-six operators that parametrise the interactions between the top-quark and the SM gauge bosons. Note that results for $p p \rightarrow t \bar{t} \gamma$ at NLO appear here for the first time, while $p p \rightarrow t \bar{t} Z$ and $e^{+} e^{-} \rightarrow t \bar{t}$ have been calculated at NLO in QCD in refs. [18, 19] in the anomalous coupling approach, albeit with the omission of the chromomagnetic dipole operator. As we will see, this operator gives a very important contribution to both the $t \bar{t} Z$ and $t \bar{t} \gamma$ processes. In addition, we also present results for the top-loop induced $H Z$ production, which involves the same operators. An important feature of our approach is that NLO predictions matched to the parton shower (PS) are provided in an automatic way. Our results are important not only because predictions are improved in accuracy and in precision, but also because NLO results can be used directly in an experimental simulation, allowing for a more dedicated investigation of all the features of any potential deviations, with possibly optimised selections and improved sensitivities to probe EFT signals. Our approach is based on the MADGRAPH5_AMC@NLO (MG5_AMC) [20] framework, and is part of the ongoing efforts of automating NLO EFT simulations for colliders [21].

The paper is organised as follows. In section 2, we present the relevant dimensionsix operators. In section 3 , we present our calculation setup. Results for the $t \bar{t} Z, t \bar{t} \gamma$, $g g \rightarrow H Z$ processes at the LHC and $t \bar{t}$ production at the ILC are given in sections $4-6$, followed by a discussion about theoretical uncertainties in section 7 . In section 8 , we discuss the sensitivity of the various processes on the operators in light of the corresponding LHC measurements. We draw our conclusions and discuss the outlook in section 9 . 


\section{Effective operators}

In an EFT approach, SM deviations are described by higher-dimensional operators. Up to dimension-six, we consider the following operators [22, 23]:

$$
\begin{aligned}
O_{\varphi Q}^{(3)} & =i \frac{1}{2} y_{t}^{2}\left(\varphi^{\dagger} \overleftrightarrow{D}_{\mu}^{I} \varphi\right)\left(\bar{Q} \gamma^{\mu} \tau^{I} Q\right) \\
O_{\varphi Q}^{(1)} & =i \frac{1}{2} y_{t}^{2}\left(\varphi^{\dagger} \overleftrightarrow{D}_{\mu} \varphi\right)\left(\bar{Q} \gamma^{\mu} Q\right) \\
O_{\varphi t} & =i \frac{1}{2} y_{t}^{2}\left(\varphi^{\dagger} \overleftrightarrow{D}_{\mu} \varphi\right)\left(\bar{t} \gamma^{\mu} t\right) \\
O_{t W} & =y_{t} g_{w}\left(\bar{Q} \sigma^{\mu \nu} \tau^{I} t\right) \tilde{\varphi} W_{\mu \nu}^{I} \\
O_{t B} & =y_{t} g_{Y}\left(\bar{Q} \sigma^{\mu \nu} t\right) \tilde{\varphi} B_{\mu \nu} \\
O_{t G} & =y_{t} g_{s}\left(\bar{Q} \sigma^{\mu \nu} T^{A} t\right) \tilde{\varphi} G_{\mu \nu}^{A}
\end{aligned}
$$

where $Q$ is the third generation left-handed quark doublet, $\varphi$ is the Higgs field, $g_{W}, g_{Y}$ and $g_{s}$ are the SM gauge coupling constants, $y_{t}$ is the top-Yukawa coupling, defined by $y_{t}=\sqrt{2} m_{t} / v$ where $v$ is the Higgs vacuum expectation value and $m_{t}$ is the pole mass (and so $y_{t}$ does not run). At lowest order in perturbation expansion, the Lagrangian is modified by these operators as follows:

$$
\Delta \mathcal{L}=\sum_{i} \frac{C_{i}}{\Lambda^{2}} O_{i}+\text { h.c. }
$$

Note that the Hermitian conjugate of each operator is added.

The above operators form a complete set that parameterises the top-quark couplings to the gluon and the electroweak gauge bosons of the SM, which could contribute at $\mathcal{O}\left(\Lambda^{-2}\right)$. In this work, we focus on their contributions to top production processes at colliders calculated at NLO in QCD. The first three operators are tree-level generated current-current operators. They modify the vector and axial-vector coupling of the top quark to the electroweak gauge bosons. The other three are dipole operators, that are more likely to be loop induced. $O_{t W}$ and $O_{t B}$ give rise to electroweak dipole moments, and $O_{t G}$ is the chromomagnetic dipole operator, relevant for the interaction of the top quark with gluons. Up to order $\Lambda^{-2}$, the cross sections and differential observables considered in this work do not receive CP-odd contributions, so in the following we assume the coefficients of $O_{t W, t B, t G}$ to be real. The three current operators are Hermitian so their coefficients are always real. The operators enter the vertices are marked out on the example Feynman diagram for the $t \bar{t} Z, t \bar{t} \gamma$ processes in figure 1 .

A complete study of the processes considered here involve more operators at dimensionsix. For example, four-fermion operators featuring top-quark pairs will also contribute to these processes. They are the same set of seven operators that contribute to top pair production as discussed in [24, 25]. Additional four-fermion operators could enter and modify the $t \bar{t} Z$ vertex through loops. In this work, we will not consider this kind of operators, postponing this to future studies. Operators involving the gauge bosons and light quarks could in principle contribute to these processes, but as they receive stringent constraints from 


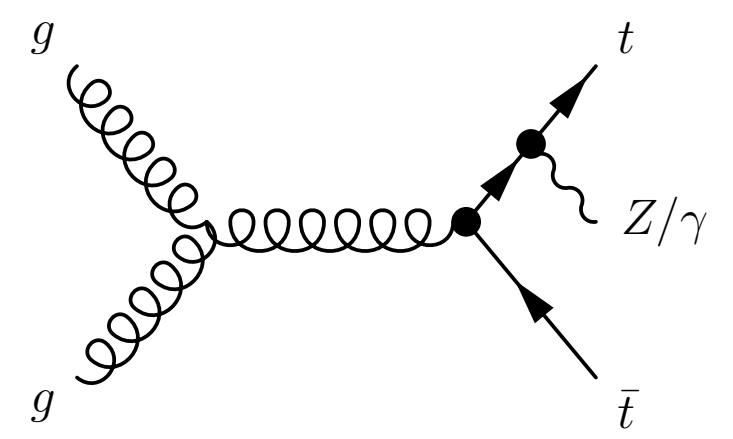

Figure 1. Example Feyman diagram for $t \bar{t} Z$ and $t \bar{t} \gamma$ production. The operators we consider can enter the $g t \bar{t} \operatorname{vertex}\left(\mathcal{O}_{t G}\right)$, the $t \bar{t} \gamma \operatorname{vertex}\left(\mathcal{O}_{t W}, \mathcal{O}_{t B}\right)$ or the $t \bar{t} Z \operatorname{vertex}\left(\mathcal{O}_{\phi t}, \mathcal{O}_{\phi Q}^{(3)}, \mathcal{O}_{\phi Q}^{(1)}, \mathcal{O}_{t W}, \mathcal{O}_{t B}\right)$.

precision observables, we consider their effect to these processes to be negligible compared to the top operators. Another operator that contributes to the $t \bar{t} Z / t \bar{t} \gamma$ processes is $O_{G}$, which would enter by modifying the gluon self-interactions. As this is not a top-quark operator, we will not consider it further here, assuming also that its contribution is sufficiently suppressed due to constraints from the accurately measured $t \bar{t}$ and dijet cross sections.

In our approach, we also take into account an additional operator, $O_{\varphi b}$ (identical to $O_{\varphi t}$ with $b$ replacing $t$ ), which does not involve a top quark, but does contribute to, for example, NLO $t \bar{t} Z$ production through a bottom loop or $b$-quarks in the initial state as well as $H Z$ production in gluon fusion through the bottom loops. We include it in this study mainly as an option to cancel the $g g Z$ chiral anomaly induced by modifications to the $t t Z$ interaction.

Various constraints can be placed on the Wilson coefficients of the top quark operators of eqs. (2.1)-(2.6) both from direct measurements and from electroweak precision measurements. For $\Lambda=1 \mathrm{TeV}$, at $95 \%$ confidence level, $C_{t G}$ is constrained from top pair production to be within the range $[-0.77,0.4]$ in ref. [26], and in ref. [16] $[-0.56,0.41]$ at leading order (LO) and $[-0.42,0.30]$ at NLO. $C_{t W}$ is constrained from $W$ helicity fractions in top-decay measurements and single top production, to be in the interval $[-0.15,1.9][27]$. The $Z \rightarrow b \bar{b}$ decay constrains the sum of $C_{\phi Q}^{(3)}+C_{\phi Q}^{(1)}$ to be [-0.026, 0.059] [28]. The other three operator coefficients, $C_{\phi Q}^{(3)}-C_{\phi Q}^{(1)}, C_{\phi t}$ and $C_{t B}$ receive indirect constraints from precision electroweak data, which lead to the following limits $[28,29]$ :

$$
\begin{aligned}
& C_{\phi Q}^{(3)}-C_{\phi Q}^{(1)}:[-3.4,7.5] \\
& C_{\phi t}:[-2.5,7] \\
& C_{t B}:[-16,43] .
\end{aligned}
$$

Note that indirect bounds should be interpreted carefully. The presented bounds here are marginalised over the $S$ and $T$ parameters, with all other operator coefficients assumed to vanish. We note here that comparable limits have been set on these operators by the recent collider based global analyses of $[11,12]$. Furthermore, RG-induced limits can also be found in [30].

Finally, let us stress that even though we work in the context of the SMEFT, the NLO calculations presented in this work can be directly used in analyses employing an 
anomalous couplings parametrisation, under the condition that $C_{t G}=0$ is assumed at all scales. In this case, operators do not mix under RG flow, and they only contribute via anomalous couplings in $t t V, b b V$ and $t b W$ vertices, and our NLO results can be translated into the anomalous coupling approach. The relations between the anomalous couplings and the effective operator coefficients are given in appendix A.

\section{Calculation setup}

Our computation is performed within the MG5_AMC framework [20], where all the elements entering the NLO computations are available automatically starting from the SMEFT Lagrangian [31-36]. NLO results can be matched to parton shower programs, such as PYTHIA8 [37] and HERWIG++ [38], through the MC@NLO [39] formalism.

Special care needs to be taken for the UV and R2 counterterms, which are required for the virtual corrections. The $\mathrm{R} 2$ terms are obtained automatically through the NLOCT package [33], and have been checked against analytical calculations. The UV counterterms depend on the renormalisation scheme. For the SM part, we use $\overline{M S}$ with five-flavor running of $\alpha_{s}$ with the top-quark subtracted at zero momentum transfer. The bottom quark mass is neglected throughout. Masses and wave-functions are renormalised on shell. The operator $O_{t G}$ gives additional contributions to the top-quark and gluon fields, as well as $\alpha_{s}$ renormalisation [16]. The operator coefficients are subtracted with the $\overline{M S}$ scheme. They are renormalised by

$$
C_{i}^{0} \rightarrow Z_{i j} C_{j}=\left(\mathbb{1}+\frac{1}{2} \Gamma(1+\varepsilon)(4 \pi)^{\varepsilon} \frac{1}{\varepsilon_{U V}} \gamma\right)_{i j} C_{j},
$$

where the anomalous dimension matrix $\gamma$ has non-zero components for the dipole operators $O_{t G}, O_{t W}$, and $O_{t B}$. The anomalous dimensions for these three operators are [14, 40-42]

$$
\gamma=\frac{2 \alpha_{s}}{\pi}\left(\begin{array}{ccc}
\frac{1}{6} & 0 & 0 \\
\frac{1}{3} & \frac{1}{3} & 0 \\
\frac{5}{9} & 0 & \frac{1}{3}
\end{array}\right) .
$$

The other operators do not have an anomalous dimension at order $\mathcal{O}\left(\alpha_{s}\right)$ due to current conservation. Results in this work are presented in terms of operators defined at the renormalisation scale, which we take as $m_{t}$ for $p p \rightarrow t \bar{t} V$ and $e^{+} e^{-} \rightarrow t \bar{t}$, and $m_{H}$ for $p p \rightarrow H Z$. If the operator coefficients are known at the new physics scale $\Lambda$, the above anomalous dimension matrix can be used to evolve them down to the renormalisation scale, to resum the large $\log \Lambda / m_{t}$ terms. Hence results presented in this work are free of such large log terms. In general, we find that NLO results cannot be approximated using the renormalisation group equations of the operators.

Operators that modify the $t t Z$ axial coupling may induce a chiral anomaly in the $g g Z$ three point function, which has an effect in $t \bar{t} Z$ and $g g \rightarrow H Z$ production. The cancellation of the anomaly depends on the details of the underlying model. To cancel this anomaly 
within the EFT framework, one option is to include the operator $O_{\phi b}$, which modifies the $b b Z$ coupling, and require

$$
C_{\phi b}=2 C_{\phi Q}^{(1)}-C_{\phi t}
$$

so that the change in $t t Z$ and $b b Z$ vertices cancel each other in the $g g Z$ function. In this work, we keep this anomaly in the calculation, and take the point of view of [43], i.e. the chiral anomaly in an effective theory is allowed, provided the corresponding gauge boson is massive. We have checked that, in either case, the numerical effect is negligible. Note that the $\mathrm{SU}(3)_{C}$ gauge is not affected, and related Ward Identities have been verified.

As a cross-check of our implementation we have compared our (LO) results with those presented in ref. [19], and have found agreement.

\section{Results for $t \bar{t} Z, t \bar{t} \gamma$ and $t \bar{t} \mu^{+} \mu^{-}$}

\subsection{Inclusive $t \bar{t} Z, t \bar{t} \gamma$ and $t \bar{t} \mu^{+} \mu^{-}$results}

In this section, we consider the inclusive $t \bar{t} Z, t \bar{t} \gamma$ and $t \bar{t} l^{+} l^{-}$cross sections including the dimension-six operators. The $t \bar{t} l^{+} l^{-}$cross section includes the contribution of off-shell photons and the interference of $t \bar{t} Z$ and $t \bar{t} \gamma^{*}$. In fact, this is the process that is experimentally accessible at the LHC, though the difference between $t \bar{t} l^{+} l^{-}$and $t \bar{t} Z$ with leptonic $Z$ decay is small for a lepton pair invariant mass close to the $Z$ boson mass.

We work up to $\mathcal{O}\left(\Lambda^{-2}\right)$, generating Feynman diagrams with at most one effective vertex. The cross section can then be expressed in the form:

$$
\sigma=\sigma_{S M}+\sum_{i} \frac{C_{i}}{(\Lambda / 1 \mathrm{TeV})^{2}} \sigma_{i}^{(1)}+\sum_{i \leq j} \frac{C_{i} C_{j}}{(\Lambda / 1 \mathrm{TeV})^{4}} \sigma_{i j}^{(2)},
$$

with the sum running over all operators in eqs. (2.1)-(2.6). Here $\sigma_{i}^{(1)}$ is the cross section of the interference of diagrams with one EFT vertex with diagrams from the SM. The cross section $\sigma_{i j}^{(2)}$, corresponds to the interference of two diagrams with one EFT vertex each or the squares of the amplitudes with one effective vertex for $i=j$.

Our implementation allows the extraction of the $\mathcal{O}\left(\Lambda^{-2}\right)$ contribution $\sigma_{i}^{(1)}$ as well as the $\mathcal{O}\left(\Lambda^{-4}\right)$ contribution $\sigma_{i j}^{(2)}$. While the latter is formally higher-order with respect to the $\mathcal{O}\left(\Lambda^{-2}\right)$ accuracy of our computation in the SMEFT, it is important for several reasons. First, as this term is of higher-order one can decide to include it without changing the accuracy of the prediction of the central value. Arguments in favour of this approach in the SMEFT have been put forward, see e.g. [44, 45]. Finally, the $\mathcal{O}\left(\Lambda^{-4}\right)$ terms are useful to associate an uncertainty to missing higher-orders in the EFT expansion. For these reasons, we quote results for $\sigma_{i i}^{(2)}$ (i.e. the squared contribution from $\mathcal{O}_{i}$ ), to either improve the central value predictions or to (partly) assess the size of the theoretical uncertainties associated to the contribution of $\mathcal{O}\left(\Lambda^{-4}\right)$ and higher terms.

In this context, we point out that the relative size of $\sigma_{i i}^{(2)}$ with respect to $\sigma_{i}^{(1)}$ cannot be used to infer the breaking down of the EFT expansion which even in the case where $\sigma_{i i}^{(2)} \gg \sigma_{i}^{(1)}$ could still be valid. One reason is that $\sigma_{i}^{(1)}$ is an interference term and various cancellations could occur accidentally. We will see this is indeed the case for several 
operators in $t \bar{t} V$ production. On the other hand, the EFT expansion in $E^{2} / \Lambda^{2}$ could still be well-behaved, or at least can be controlled by applying kinematic cuts on the total energy $E$ of the process. In this respect, as we were mentioning above, a legitimate and motivated way to proceed is to always include both interference and squared contributions, and separately estimate the theoretical error due to missing dimension-eight operators. Another interesting possibility is in the presence of "strong interactions", i.e. when $C_{i}^{2} \frac{E^{4}}{\Lambda^{4}}>$ $C_{i} \frac{E^{2}}{\Lambda^{2}}>1>\frac{E^{2}}{\Lambda^{2}}$. In this case, the squared contribution dominates over the interference one, without invalidating the $E^{2} / \Lambda^{2}$ expansion, which is parametrically independent of the size of the coefficients. In a phenomenological analysis and in a global fit, all such cases should be always kept in mind and carefully analysed on the basis of the resulting bounds on the $C_{i}$ 's. As the main goal of this paper is to present a framework to perform calculations in the SMEFT at NLO accuracy and study the results for the neutral top interactions, we do not discuss any further the issue related to the size of the coefficients and the validity conditions of the EFT itself. On the other hand, we stress that our implementation/framework can provide the elements necessary to make a detailed study. For example, we present the full results at $\mathcal{O}\left(\Lambda^{-2}\right)$, characterised by $\sigma_{i}^{(1)}$, together with $\sigma_{i i}^{(2)}$ as an estimation of uncertainties due to neglecting all $\sigma_{i j}^{(2)}$ terms. Note that if necessary, any $\sigma_{i j}^{(2)}$ term can be also computed.

In practice, to extract the values of $\sigma_{i}^{(1)}$, we set one of the $C_{i}$ coefficients to \pm 1 and all the others to zero. Using the two values and the SM cross-section, we can extract $\sigma_{i}^{(1)}$, as well as $\sigma_{i i}^{(2)}$, the contribution of the $\mathcal{O}\left(\Lambda^{-2}\right)$ amplitudes squared. In order to improve the statistical significance of the interference for the operators where the interference is small, we find the value of $C_{i}$ which maximises it compared to the total cross-section and use that value for the runs instead of $C_{i}= \pm 1$.

The results are obtained using the 5-flavour scheme, with the MSTW2008 [46] parton distribution functions. The input parameters are:

$$
\begin{aligned}
m_{t} & =173.3 \mathrm{GeV}, & m_{Z} & =91.1876 \mathrm{GeV}, \\
\alpha_{E W}^{-1} & =127.9, & G_{F} & =1.16637 \times 10^{-5} \mathrm{GeV}^{-2} .
\end{aligned}
$$

The renormalisation and factorisation scales are fixed to $\mu_{R}=\mu_{F}=\mu=m_{t}$. For a detailed discussion of scale choices for the $t \bar{t} V$ processes see [47]. Scale variations are obtained by independently setting $\mu_{R}$ and $\mu_{F}$ to $\mu / 2, \mu$ and $2 \mu$, obtaining nine $\left(\mu_{R}, \mu_{F}\right)$ combinations. For the $t \bar{t} Z$ process no cuts are applied on the final state particles and no $Z$ or top decays are considered, while for $t \bar{t} \gamma, p_{T}(\gamma)>20 \mathrm{GeV}$ is required. We employ the photon isolation criterium of ref. [48] with a radius of 0.4 . Finally, for the $t \bar{t} \mu^{+} \mu^{-}$process a cut of $10 \mathrm{GeV}$ is set on the minimum invariant mass of the lepton pair.

The SM predictions at $\mathrm{LO}$ and $\mathrm{NLO}$ in $\mathrm{QCD}^{1}$ for the processes considered here are summarised as a reference in table 1, where uncertainties from scale variation, PDF uncertainties, and the $K$-factors are shown for the LHC at 8 and $13 \mathrm{TeV}$. The scale uncertainties are significantly reduced at NLO. The PDF uncertainties are small compared to the scale uncertainties even at NLO and therefore we will not consider them any further.

\footnotetext{
${ }^{1}$ Note that the SM results for the $t \bar{t} Z$ process have been presented at NLO in the QCD and electroweak coupling expansion in [49].
} 


\begin{tabular}{|c|c|c|c|c|}
\hline SM & {$[\mathrm{fb}]$} & $t \bar{t} Z$ & $t \bar{t} \gamma$ & $t \bar{t} \mu^{+} \mu^{-}$ \\
\hline \multirow[t]{3}{*}{$8 \mathrm{TeV}$} & $\sigma_{S M, L O}$ & $207.0_{-26.8 \%}^{+41.4 \%}+2.4 \%$ & $604.0_{-25.6 \%}^{+38.8 \%}+2.2 \%$ & $8.779_{-26.6 \%}^{+40.9 \%}{ }_{-2.4 \%}^{+2.4 \%}$ \\
\hline & $\sigma_{S M, N L O}$ & $226.5_{-11.2 \%}^{+6.7 \%}{ }_{-3.2 \%}^{+2.8 \%}$ & $777_{-13.7 \%}^{+13.4 \%}{ }_{-2.4 \%}^{+2.1 \%}$ & $9.827_{-11.5 \%}^{+7.7 \%}{ }_{-2.9 \%}^{+2.6 \%}$ \\
\hline & $K$-factor & 1.09 & 1.29 & 1.12 \\
\hline \multirow[t]{3}{*}{$13 \mathrm{TeV}$} & $\sigma_{S M, L O}$ & $761.8_{-25.2 \%}^{+37.8 \%}+2.2 \%$ & $1998.0_{-24.2 \%}^{+35.5 \%}+1.8 \%$ & $31.67_{-25.1 \%}^{+37.4 \%}+2.2 .1 \%$ \\
\hline & $\sigma_{S M, N L O}$ & $879_{-10.9 \%}^{+8.0 \%}{ }_{-2.5 \%}^{+2.0 \%}$ & $2719_{-13.5 \%}^{+14.2 \%}{ }_{-1.9 \%}^{+1.6 \%}$ & $37.51_{-11.3 \%}^{+9.1 \%}{ }_{-2.4 \%}^{+2.0 \%}$ \\
\hline & $K$-factor & 1.15 & 1.36 & 1.18 \\
\hline
\end{tabular}

Table 1. SM cross sections (in fb) for $t \bar{t} Z, t \bar{t} \gamma, t \bar{t} \mu^{+} \mu^{-}$production at the LHC at $\sqrt{s}=8 \mathrm{TeV}$ and $\sqrt{s}=13 \mathrm{TeV}$. The first percentage corresponds to scale variations and the second to PDF uncertainties.

Inclusive cross section results for $t \bar{t} Z$ production at the $\mathrm{LHC}$ at 8 and $13 \mathrm{TeV}$ for the different operators are shown in tables 2 and 3 . We include the LO and NLO results for $\sigma_{i}^{(1)}$ and $\sigma_{i i}^{(2)}$, the corresponding $K$-factors, the ratio of the dimension-six contribution over the $\mathrm{SM}$ and the ratio of the squared $\mathcal{O}\left(\Lambda^{-4}\right)$ contributions over the $\mathcal{O}\left(\Lambda^{-2}\right)$ one. Statistical uncertainties are not shown unless they are comparable to the scale uncertainties. The scale uncertainties are significantly reduced at NLO similarly to the SM predictions. We note that the ratios over the SM are significantly less sensitive to scale variations compared to the cross-section numbers.

In the tables, we include the $\mathcal{O}_{\phi Q}^{(3)}$ operator but not $\mathcal{O}_{\phi Q}^{(1)}$. Results for these two operators differ by a sign at $\mathcal{O}\left(\Lambda^{-2}\right)$ and are identical at $\mathcal{O}\left(\Lambda^{-4}\right){ }^{2}$. Similarly at $\mathcal{O}\left(\Lambda^{-4}\right)$ the contributions of $\mathcal{O}_{\phi Q}^{(3)}$ and $\mathcal{O}_{\phi t}$ are identical. This can be traced back to the way the operators modify the $t t Z$ vertex as shown in eq. (A.1). Similarly we do not include the results for $\mathcal{O}_{t B}$, as they can be obtained from those of $\mathcal{O}_{t W}$ by multiplying by a factor of $-\tan ^{2} \theta_{w}$ (and $\tan ^{4} \theta_{w}$ for the squared contributions).

The largest contribution is given by the chromomagnetic operator both at 8 and $13 \mathrm{TeV}$, with $\sigma_{i}^{(1)}$ reaching almost $40 \%$ of the SM. We find that while $\mathcal{O}_{\phi Q}^{(3)}$ and $\mathcal{O}_{\phi t}$ give contributions of $6-10 \%$ of the SM for $C_{i}=1, \mathcal{O}_{t W}$ and consequently $\mathcal{O}_{t B}$ give extremely small contributions reaching at most the per mille level. While the NLO predictions have significantly reduced theoretical uncertainties, we find that the various ratios of cross-sections considered are generally stable with respect to QCD corrections (apart from $\mathcal{O}_{t W}$ ), and also suffer from much smaller scale uncertainties compared to the cross-sections. This fact can be exploited to extract information on the Wilson coefficients. The theoretical errors due to neglecting squared operator contributions $\sigma_{i i}^{(2)}$ are characterised by the last two rows in the table. The results indicate that for coefficients of order one, neglecting squared

\footnotetext{
${ }^{2}$ This is only approximately true at the cross-section level. There is a small contribution from the $b b Z$ vertex which spoils the minus sign relation between the two operators. The $b b Z$ vertex contributes as we are working in the 5 -flavour scheme. Nevertheless this contribution is in practice numerically negligible and therefore the two operators give opposite contributions at $\mathcal{O}\left(\Lambda^{-2}\right)$.
} 


\begin{tabular}{|ccccc|}
\hline $8 \mathrm{TeV}$ & $\mathcal{O}_{t G}$ & $\mathcal{O}_{\phi Q}^{(3)}$ & $\mathcal{O}_{\phi t}$ & $\mathcal{O}_{t W}$ \\
\hline$\sigma_{i, L O}^{(1)}$ & $76.1_{-27.1 \%}^{+41.9 \%}$ & $18.6_{-28.6 \%}^{+45.2 \%}$ & $12.5_{-28.3 \%}^{+44.6 \%}$ & $0.077(8)_{-43.2 \%}^{+46.6 \%}$ \\
$\sigma_{i, N L O}^{(1)}$ & $78.1_{-10.0 \%}^{+4.1 \%}$ & $20.8_{-11.5 \%}^{+5.6 \%}$ & $13.5_{-10.7 \%}^{+4.9 \%}$ & $-0.32(2)_{-67.3 \%}^{+39.1 \%}$ \\
$K$-factor & 1.03 & 1.12 & 1.08 & -4.2 \\
$\sigma_{i i, L O}^{(2)}$ & $39.9_{-31.8 \%}^{+53.6 \%}$ & $0.73(2)_{-28.8 \%}^{+45.2 \%}$ & $0.73(2)_{-28.8 \%}^{+46.3 \%}$ & $4.14_{-30.7 \%}^{+50.1 \%}$ \\
$\sigma_{i i, N L O}^{(2)}$ & $39.8_{-9.4 \%}^{+4.7 \%}$ & $0.8(2)_{-9.1 \%}^{+5.4 \%}$ & $0.8(2)_{-8.3 \%}^{+7.4 \%}$ & $4.81_{-12.5 \%}^{+6.2 \%}$ \\
$\sigma_{i, L O}^{(1)} / \sigma_{S M, L O}$ & $0.368_{-0.4 \%}^{+0.4 \%}$ & $0.0899_{-2.5 \%}^{+2.7 \%}$ & $0.0604_{-2.0 \%}^{+2.3 \%}$ & $0.00037(4)_{-42.5 \%}^{+33.6 \%}$ \\
$\sigma_{i, N L O}^{(1)} / \sigma_{S M, N L O}$ & $0.345_{-2.8 \%}^{+1.3 \%}$ & $0.0918_{-1.0 \%}^{+0.6 \%}$ & $0.0595_{-2.3 \%}^{+0.8 \%}$ & $-0.0014(1)_{-56.8 \%}^{+31.4 \%}$ \\
$\sigma_{i i, L O}^{(2)} / \sigma_{i, L O}^{(1)}$ & $0.524_{-6.5 \%}^{+8.2 \%}$ & $0.039(1)_{-0.5 \%}^{+0.3 \%}$ & $0.058(2)_{-0.7 \%}^{+1.2 \%}$ & $54(6)_{-29.1 \%}^{+84.7 \%}$ \\
$\sigma_{i i, N L O}^{(2)} / \sigma_{i, N L O}^{(1)}$ & $0.509_{-8.4 \%}^{+1.4 \%}$ & $0.037(8)_{-4.5 \%}^{+2.7 \%}$ & $0.06(1)_{-5.9 \%}^{+3.2 \%}$ & $-15(1)_{-43.5 \%}^{+36.9 \%}$ \\
\hline
\end{tabular}

Table 2. Cross sections (in fb) for $t \bar{t} Z$ production at the $\mathrm{LHC}$ at $\sqrt{s}=8 \mathrm{TeV}$ for the different dimension-six operators. Percentages correspond to scale uncertainties. Integration errors are shown in brackets if these are comparable in size to the scale uncertainties.

\begin{tabular}{|ccccc|}
\hline $13 \mathrm{TeV}$ & $\mathcal{O}_{t G}$ & $\mathcal{O}_{\phi Q}^{(3)}$ & $\mathcal{O}_{\phi t}$ & $\mathcal{O}_{t W}$ \\
\hline$\sigma_{i, L O}^{(1)}$ & $286.7_{-25.5 \%}^{+38.2 \%}$ & $78.3_{-26.6 \%}^{+40.4 \%}$ & $51.6_{-26.4 \%}^{+40.1 \%}$ & $-0.20(3)_{-230.0 \%}^{+88.0 \%}$ \\
$\sigma_{i, N L O}^{(1)}$ & $310.5_{-9.7 \%}^{+5.4 \%}$ & $90.6_{-11.0 \%}^{+7.1 \%}$ & $57.5_{-10.3 \%}^{+5.8 \%}$ & $-1.7(2)_{-49.1 \%}^{+31.3 \%}$ \\
$K$-factor & 1.08 & 1.16 & 1.11 & 8.5 \\
$\sigma_{i i, L O}^{(2)}$ & $258.5_{-30.4 \%}^{+49.7 \%}$ & $2.8(1)_{-26.9 \%}^{+39.7 \%}$ & $2.9(1)_{-26.7 \%}^{+39.7 \%}$ & $20.9_{-28.3 \%}^{+44.3 \%}$ \\
$\sigma_{i i, N L O}^{(2)}$ & $244.5_{-8.1 \%}^{+4.2 \%}$ & $3.8(3)_{-14.4 \%}^{+13.2 \%}$ & $3.9(3)_{-14.6 \%}^{+13.8 \%}$ & $24.2_{-11.2 \%}^{+6.2 \%}$ \\
$\sigma_{i, L O}^{(1)} / \sigma_{S M, L O}$ & $0.376_{-0.3 \%}^{+0.3 \%}$ & $0.103_{-1.8 \%}^{+1.9 \%}$ & $0.0677_{-1.6 \%}^{+1.7 \%}$ & $-0.00026(4)_{-167.2 \%}^{+89.5 \%}$ \\
$\sigma_{i, N L O}^{(1)} / \sigma_{S M, N L O}$ & $0.353_{-2.4 \%}^{+1.3 \%}$ & $0.103_{-0.8 \%}^{+0.7 \%}$ & $0.0654_{-2.1 \%}^{+1.1 \%}$ & $-0.0020(2)_{-38.0 \%}^{+22.9 \%}$ \\
$\sigma_{i i, L O}^{(2)} / \sigma_{i, L O}^{(1)}$ & $0.902_{-6.7 \%}^{+8.4 \%}$ & $0.036(1)_{-1.1 \%}^{+0.2 \%}$ & $0.056(2)_{-0.3 \%}^{+0.6 \%}$ & $-104(16)_{-815.2 \%}^{+60.8 \%}$ \\
$\sigma_{i i, N L O}^{(2)} / \sigma_{i, N L O}^{(1)}$ & $0.787_{-12.8 \%}^{+3.3 \%}$ & $0.042(4)_{-3.9 \%}^{+5.6 \%}$ & $0.067(6)_{-4.8 \%}^{+7.6 \%}$ & $-14(1)_{-29.1 \%}^{+29.0 \%}$ \\
\hline
\end{tabular}

Table 3. Cross sections (in fb) for $t \bar{t} Z$ production at the $\mathrm{LHC}$ at $\sqrt{s}=13 \mathrm{TeV}$ for the different dimension-six operators. Percentages correspond to scale uncertainties. Integration errors are shown in brackets if these are comparable in size to the scale uncertainties.

contributions is safe for all operators except for $\mathcal{O}_{t B}$ and $\mathcal{O}_{t W}$. When placing limits, this assessment should be done for the interval of where the limits are placed.

We note here the extremely small contribution of the $\mathcal{O}_{t W}$ operator, which also leads to larger statistical uncertainties as it is currently not possible to compute the interference independently of the other two contributions. In this case, the impact of the EFT amplitude squared is much larger than its interference with the SM. The small size of the 


\begin{tabular}{|c|c|c|c|c|c|}
\hline $8 \mathrm{TeV}$ & $\mathcal{O}_{t G}$ & $\mathcal{O}_{t B}$ & $13 \mathrm{TeV}$ & $\mathcal{O}_{t G}$ & $\mathcal{O}_{t B}$ \\
\hline$\sigma_{i, L O}^{(1)}$ & $171.5_{-25.6 \%}^{+38.6 \%}$ & $5.36_{-27.2 \%}^{+41.8 \%}$ & & $564.6_{-24.1 \%}^{+35.4 \%}$ & $19.5_{-24.9 \%}^{+36.7 \%}$ \\
\hline$\sigma_{i, N L O}^{(1)}$ & $218.9_{-13.6 \%}^{+13.3 \%}$ & $5.85_{-9.9 \%}^{+5.9 \%}$ & & $765_{-13.4 \%}^{+14.0 \%}$ & $19.6_{-6.9 \%}^{+4.3 \%}$ \\
\hline$K$-factor & 1.28 & 1.09 & & 1.35 & 1.01 \\
\hline$\sigma_{i i, L O}^{(2)}$ & $29.8_{-27.8 \%}^{+43.5 \%}$ & $1.98_{-29.6 \%}^{+47.5 \%}$ & & $120.6_{-26.2 \%}^{+39.8 \%}$ & $9.14_{-27.4 \%}^{+42.3 \%}$ \\
\hline$\sigma_{i i, N L O}^{(2)}$ & $39.2_{-14.4 \%}^{+13.1 \%}$ & $2.36_{-12.6 \%}^{+7.0 \%}$ & & $160.4_{-13.5 \%}^{+12.6 \%}$ & $10.7_{-11.2 \%}^{+6.7 \%}$ \\
\hline$\sigma_{i, L O}^{(1)} / \sigma_{S M, L O}$ & $0.284_{-0.1 \%}^{+0.04 \%}$ & $0.00888_{-2.2 \%}^{+2.3 \%}$ & & $0.283_{-0.1 \%}^{+0.1 \%}$ & $0.00973_{-1.0 \%}^{+0.9 \%}$ \\
\hline$\sigma_{i, N L O}^{(1)} / \sigma_{S M, N L O}$ & $0.282_{-0.2 \%}^{+0.13 \%}$ & $0.0075(1)_{-8.8 \%}^{+4.4 \%}$ & & $0.281_{-0.1 \%}^{+0.1 \%}$ & $0.0072(1)_{-11.9 \%}^{+7.5 \%}$ \\
\hline$\sigma_{i i, L O}^{(2)} / \sigma_{i, L O}^{(1)}$ & $0.174_{-3.0 \%}^{+3.5 \%}$ & $0.370_{-3.3 \%}^{+4.0 \%}$ & & $0.214_{-2.8 \%}^{+3.3 \%}$ & $0.470_{-3.4 \%}^{+4.1 \%}$ \\
\hline$\sigma_{i i, N L O}^{(2)} / \sigma_{i, N L O}^{(1)}$ & $0.179_{-0.9 \%}^{+0.5 \%}$ & $0.404(7)_{-3.0 \%}^{+3.5 \%}$ & & $0.201_{-1.3 \%}^{+1.1 \%}$ & $0.55(1)_{-4.6 \%}^{+6.1 \%}$ \\
\hline
\end{tabular}

Table 4. Cross sections (in fb) for $t \bar{t} \gamma$ production at the LHC at $\sqrt{s}=8 \mathrm{TeV}$ and $\sqrt{s}=13 \mathrm{TeV}$ for the different dimension-six operators. Percentages correspond to scale uncertainties. Integration errors are shown in brackets if these are comparable in size to the scale uncertainties. A $p_{T}(\gamma)>$ $20 \mathrm{GeV}$ cut is imposed.

interference is a result of various effects. The most important reason is that the dipole interaction, $\sigma^{\mu \nu} q_{\nu}$, involves the momentum of the $Z$ boson, and leads to a suppression because the $Z$ tends to be soft in $t \bar{t} Z$ production at the LHC. The same is true also for the $t \bar{t} \gamma$ production, as we will see. By crossing $\gamma$ and $g$, we have explicitly checked that in the process $g \gamma \rightarrow t \bar{t} g$ this suppression effect becomes an enhancement, as a large momentum for $\gamma$ is guaranteed in the initial state. Apart from this, an additional suppression occurs due to an accidental cancellation between the contributions of the $g g$ and $q \bar{q}$ channels, as they are similar in size but come with an opposite sign. This cancellation leads to a final result that is an order of magnitude smaller than the individual contributions. Finally, an additional reason could be that the $O_{t W}$ vertex does not produce the $Z$ boson in its longitudinal state, which is expected to dominate if it has large momentum.

Finally, comparing 8 and $13 \mathrm{TeV}$ we notice a small increase in the $K$-factors. The ratios of the $\mathcal{O}\left(\Lambda^{-2}\right)$ terms over the SM do not change significantly. For $\mathcal{O}_{t G}$ we notice a significant increase of the ratio $\mathcal{O}\left(\Lambda^{-4}\right)$ over $\mathcal{O}\left(\Lambda^{-2}\right)$ as the $\mathcal{O}\left(\Lambda^{-4}\right)$ contribution grows rapidly with energy, as will be evident also in the differential distributions.

The corresponding $t \bar{t} \gamma$ results are shown in table 4 . In this case, a minimum cut of $20 \mathrm{GeV}$ is set on the transverse momentum of the photon. We note that here only three operators contribute: $\mathcal{O}_{t G}, \mathcal{O}_{t W}$ and $\mathcal{O}_{t B}$. For this process, $\mathcal{O}_{t W}$ and $\mathcal{O}_{t B}$ are indistinguishable and therefore only $\mathcal{O}_{t B}$ is included in the table. The $K$-factors in this process are larger than those of $t \bar{t} Z$, reaching 1.3 for the SM and $\mathcal{O}_{t G}$ operator but lower for $\mathcal{O}_{t B}$. This is due to the soft and collinear configurations between the photon and the additional jet at NLO, which however cannot happen if the photon is emitted from an $\mathcal{O}_{t B}$ vertex. 


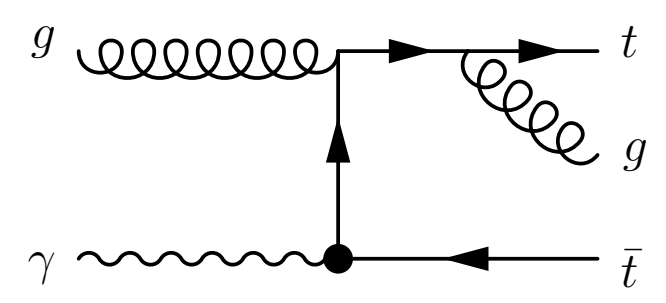

Figure 2. An example Feynman diagram for the crossed process $g \gamma \rightarrow t \bar{t} g$. The $t \bar{t} \gamma$ vertex, marked with a blob, has generally a higher momentum transfer here than in $t \bar{t} \gamma$ production.

Similar conclusions to the $t \bar{t} Z$ can be drawn for $t \bar{t} \gamma$ regarding the operator contributions. The chromomagnetic operator contributes the most. Neglecting squared contributions is safe for $C_{i} \lesssim 1$, at both 8 and $13 \mathrm{TeV}$, but starts to become questionable (and therefore the corresponding uncertainty is increased) as the coefficients reach order of a few, with the relative contribution of $\sigma_{i i}^{(2)}$ increasing from 8 to $13 \mathrm{TeV}$. The contribution of the $\mathcal{O}_{t W}$ and $\mathcal{O}_{t B}$ operators are $1 \%$ of the $\mathrm{SM}$ and significantly smaller than the $\mathcal{O}_{t G}$ one. While the $\mathcal{O}_{t W}$ and $\mathcal{O}_{t G}$ operators lead to the same structure in the $t t \gamma$ and $t t g$ vertices respectively, similar to $t t Z$ production, the effect of $\mathcal{O}_{t W}$ on the $g g \rightarrow t \bar{t} \gamma$ amplitude at typical LHC energies is suppressed compared with that of $\mathcal{O}_{t G}$. By examining the crossed amplitude, $g \gamma \rightarrow t \bar{t} g$, illustrated in figure 2, we see that the two operators give contributions of the same order, as they both enter in the production side of the process and more momentum passes through the EFT vertices. We also note here that the $K$-factors for the operators are not the same as those as for the SM contribution which implies that combining the SM $K$-factor and LO EFT predictions does not provide an accurate prediction for the EFT contribution at NLO in QCD.

We next examine $t \bar{t} l^{+} l^{-}$. For an invariant mass of the lepton pair around the $Z$ mass, this process is dominated by $t \bar{t} Z$ with leptonically decaying $Z$, the mode that the ATLAS and CMS experiments at the LHC are most sensitive to. Generally it also includes the contribution of $t \bar{t} \gamma^{*}$. As the EFT operators we study do not enter the vertices connected to leptons, we restrict our attention to $t \bar{t} \mu^{+} \mu^{-}{ }^{3}$ We collect the results for $t \bar{t} \mu^{+} \mu^{-}$at LO and $\mathrm{NLO}$ at 8 and $13 \mathrm{TeV}$ in tables 5 and 6 . In this case, the photon and $Z$ amplitudes and their interference is included. For the $t \bar{t} \mu^{+} \mu^{-}$results, the scale and PDF choices are identical to those for the inclusive $t \bar{t} Z / t \bar{t} \gamma$ processes. A lower cut of $10 \mathrm{GeV}$ is imposed on the invariant mass of the lepton pair. No other cuts are imposed on the leptons.

All six operators contribute to this process. Results for $\mathcal{O}_{\phi Q}^{(1)}$ differ from those of $\mathcal{O}_{\phi Q}^{(3)}$ by a sign at $\mathcal{O}\left(\Lambda^{-2}\right)$ and are identical at $\mathcal{O}\left(\Lambda^{-4}\right)$, therefore we show only one of the two. The cross-section is dominated by the region close to the $Z$-mass peak and therefore the $K$-factors and relative contributions of the operators are similar to those of the $t \bar{t} Z$ process. The chromomagnetic operator contributes at the $35 \%$ level, while the other three current operators give a contribution at the $4-7 \%$ level.

\footnotetext{
${ }^{3}$ We note here that a contribution from 4 -fermion operators describing the $t \bar{t} \mu^{+} \mu^{-}$interaction enter in this process in the off-peak regions. As the main contribution comes from the $Z$-peak we postpone the study of these operators to future work.
} 


\begin{tabular}{|cccccc|}
\hline $8 \mathrm{TeV}$ & $\mathcal{O}_{t G}$ & $\mathcal{O}_{\phi Q}^{(3)}$ & $\mathcal{O}_{\phi t}$ & $\mathcal{O}_{t B}$ & $\mathcal{O}_{t W}$ \\
\hline$\sigma_{i, L O}^{(1)}$ & $3.07_{-26.9 \%}^{+41.5 \%}$ & $0.613_{-28.6 \%}^{+45.2 \%}$ & $0.413_{-28.3 \%}^{+44.6 \%}$ & $0.0101_{-27.6 \%}^{+43.2 \%}$ & $0.0121(6)_{-21.5 \%}^{+29.2 \%}$ \\
$\sigma_{i, N L O}^{(1)}$ & $3.21_{-10.4 \%}^{+5.1 \%}$ & $0.683_{-11.3 \%}^{+5.4 \%}$ & $0.447_{-10.9 \%}^{+4.8 \%}$ & $0.012(1)_{-12.2 \%}^{+8.9 \%}$ & $-0.003(2)_{-205.9 \%}^{+113.9 \%}$ \\
$K$-factor & 1.05 & 1.11 & 1.08 & 1.2 & -0.3 \\
$\sigma_{i i, L O}^{(2)}$ & $1.42_{-31.6 \%}^{+52.9 \%}$ & $0.0238_{-28.7 \%}^{+45.2 \%}$ & $0.0234_{-28.7 \%}^{+45.8 \%}$ & $0.0213_{-30.6 \%}^{+49.8 \%}$ & $0.147_{-30.7 \%}^{+50.1 \%}$ \\
$\sigma_{i i, N L O}^{(2)}$ & $1.41_{-9.7 \%}^{+4.5 \%}$ & $0.0275_{-11.7 \%}^{+6.4 \%}$ & $0.0259_{-11.4 \%}^{+5.0 \%}$ & $0.0249_{-12.6 \%}^{+6.5 \%}$ & $0.171_{-12.5 \%}^{+6.3 \%}$ \\
$\sigma_{i, L O}^{(1)} / \sigma_{S M, L O}$ & $0.350_{-0.4 \%}^{+0.4 \%}$ & $0.0698_{-2.8 \%}^{+3.1 \%}$ & $0.0470_{-2.3 \%}^{+2.6 \%}$ & $0.00115_{-1.7 \%}^{+1.6 \%}$ & $0.0014(1)_{-8.4 \%}^{+6.9 \%}$ \\
$\sigma_{i, N L O}^{(1)} / \sigma_{S M, N L O}$ & $0.327_{-2.4 \%}^{+1.2 \%}$ & $0.0695_{-2.3 \%}^{+1.0 \%}$ & $0.0455_{-2.8 \%}^{+1.3 \%}$ & $0.0012(1)_{-1.5 \%}^{+2.0 \%}$ & $-0.0004(2)_{-184.1 \%}^{+115.7 \%}$ \\
$\sigma_{i i, L O}^{(2)} / \sigma_{i, L O}^{(1)}$ & $0.461_{-6.5 \%}^{+8.1 \%}$ & $0.0388_{-0.1 \%}^{+0.0 \%}$ & $0.0567_{-0.7 \%}^{+0.8 \%}$ & $2.11(5)_{-4.1 \%}^{+5.2 \%}$ & $12.2(6)_{-11.7 \%}^{+16.3 \%}$ \\
$\sigma_{i i, N L O}^{(2)} / \sigma_{i, N L O}^{(1)}$ & $0.440_{-8.3 \%}^{+1.7 \%}$ & $0.0403(8)_{-0.7 \%}^{+1.0 \%}$ & $0.058(2)_{-0.6 \%}^{+0.4 \%}$ & $2.1(1)_{-2.8 \%}^{+2.6 \%}$ & $-49(23)_{-332.3 \%}^{+730.1 \%}$ \\
\hline
\end{tabular}

Table 5. Cross sections (in fb) for $t \bar{t} \mu^{+} \mu^{-}$production at the LHC at $\sqrt{s}=8 \mathrm{TeV}$ for the different dimension-six operators. An $m(\ell \ell)>10 \mathrm{GeV}$ cut is applied to the lepton pair. Percentages correspond to scale uncertainties. Integration errors are shown in brackets if these are comparable in size to the scale uncertainties.

\begin{tabular}{|cccccc|}
\hline $13 \mathrm{TeV}$ & $\mathcal{O}_{t G}$ & $\mathcal{O}_{\phi Q}^{(3)}$ & $\mathcal{O}_{\phi t}$ & $\mathcal{O}_{t B}$ & $\mathcal{O}_{t W}$ \\
\hline$\sigma_{i, L O}^{(1)}$ & $11.28_{-25.2 \%}^{+3.8 \%}$ & $2.584_{-26.6 \%}^{+40.4 \%}$ & $1.701_{-26.4 \%}^{+40.1 \%}$ & $0.034(1)_{-25.1 \%}^{+36.9 \%}$ & $0.025(3)_{-24.8 \%}^{+29.4 \%}$ \\
$\sigma_{i, N L O}^{(1)}$ & $12.57_{-10.3 \%}^{+6.7 \%}$ & $2.976_{-10.8 \%}^{+6.7 \%}$ & $1.891_{-10.1 \%}^{+5.4 \%}$ & $0.046(2)_{-12.7 \%}^{+13.0 \%}$ & $-0.042(9)_{-73.2 \%}^{+44.6 \%}$ \\
$K$-factor & 1.11 & 1.15 & 1.11 & 1.3 & -1.7 \\
$\sigma_{i i, L O}^{(2)}$ & $8.957_{-30.2 \%}^{+49.3 \%}$ & $0.101_{-26.6 \%}^{+40.4 \%}$ & $0.0998_{-26.6 \%}^{+40.6 \%}$ & $0.1073_{-28.3 \%}^{+44.3 \%}$ & $0.745_{-28.4 \%}^{+44.4 \%}$ \\
$\sigma_{i i, N L O}^{(2)}$ & $8.49_{-7.4 \%}^{+4.1 \%}$ & $0.1168_{-11.0 \%}^{+7.1 \%}$ & $0.112(3)_{-10.0 \%}^{+5.5 \%}$ & $0.1231_{-11.0 \%}^{+6.2 \%}$ & $0.851_{-11.0 \%}^{+5.9 \%}$ \\
$\sigma_{i, L O}^{(1)} / \sigma_{S M, L O}$ & $0.356_{-0.2 \%}^{+0.3 \%}$ & $0.0816_{-2.0 \%}^{+2.2 \%}$ & $0.0537_{-1.8 \%}^{+2.0 \%}$ & $0.00108(3)_{-0.5 \%}^{+0.3 \%}$ & $0.0008(1)_{-16.1 \%}^{+12.7 \%}$ \\
$\sigma_{i, N L O}^{(1)} / \sigma_{S M, N L O}$ & $0.335_{-2.2 \%}^{+1.2 \%}$ & $0.0793_{-2.2 \%}^{+1.1 \%}$ & $0.0504_{-3.5 \%}^{+1.5 \%}$ & $0.0012(1)_{-1.6 \%}^{+3.6 \%}$ & $-0.0011(2)_{-58.7 \%}^{+37.6 \%}$ \\
$\sigma_{i i, L O}^{(2)} / \sigma_{i, L O}^{(1)}$ & $0.794_{-6.7 \%}^{+8.4 \%}$ & $0.0390_{-0.02 \%}^{+0.03 \%}$ & $0.0586_{-0.4 \%}^{+0.5 \%}$ & $3.15(9)_{-4.6 \%}^{+5.5 \%}$ & $29(4)_{-15.2 \%}^{+25.3 \%}$ \\
$\sigma_{i i, N L O}^{(2)} / \sigma_{i, N L O}^{(1)}$ & $0.676_{-13.3 \%}^{+3.6 \%}$ & $0.0393_{-0.2 \%}^{+0.3 \%}$ & $0.059(1)_{-0.2 \%}^{+0.2 \%}$ & $2.7(1)_{-6.8 \%}^{+2.1 \%}$ & $-20(5)_{-60.7 \%}^{+39.2 \%}$ \\
\hline
\end{tabular}

Table 6. Cross sections (in fb) for $t \bar{t} \mu^{+} \mu^{-}$production at the LHC at $\sqrt{s}=13 \mathrm{TeV}$ for the different dimension-six operators. An $m(\ell \ell)>10 \mathrm{GeV}$ cut is applied to the lepton pair. Percentages correspond to scale uncertainties. Integration errors are shown in brackets if these are comparable in size to the scale uncertainties.

The contributions of $\mathcal{O}_{t W}$ and $\mathcal{O}_{t B}$ at $\mathcal{O}\left(\Lambda^{-2}\right)$ are at the per mille level and subdominant compared to the $\mathcal{O}\left(\Lambda^{-4}\right)$ contributions. Effectively this means that with our method of extracting the interference contribution we are always very limited statistically. Even maximising the interference contribution by choosing the appropriate value of the coefficient is not enough to give us good statistics, in particular at NLO which is evident in the quoted statistical and scale uncertainties. 

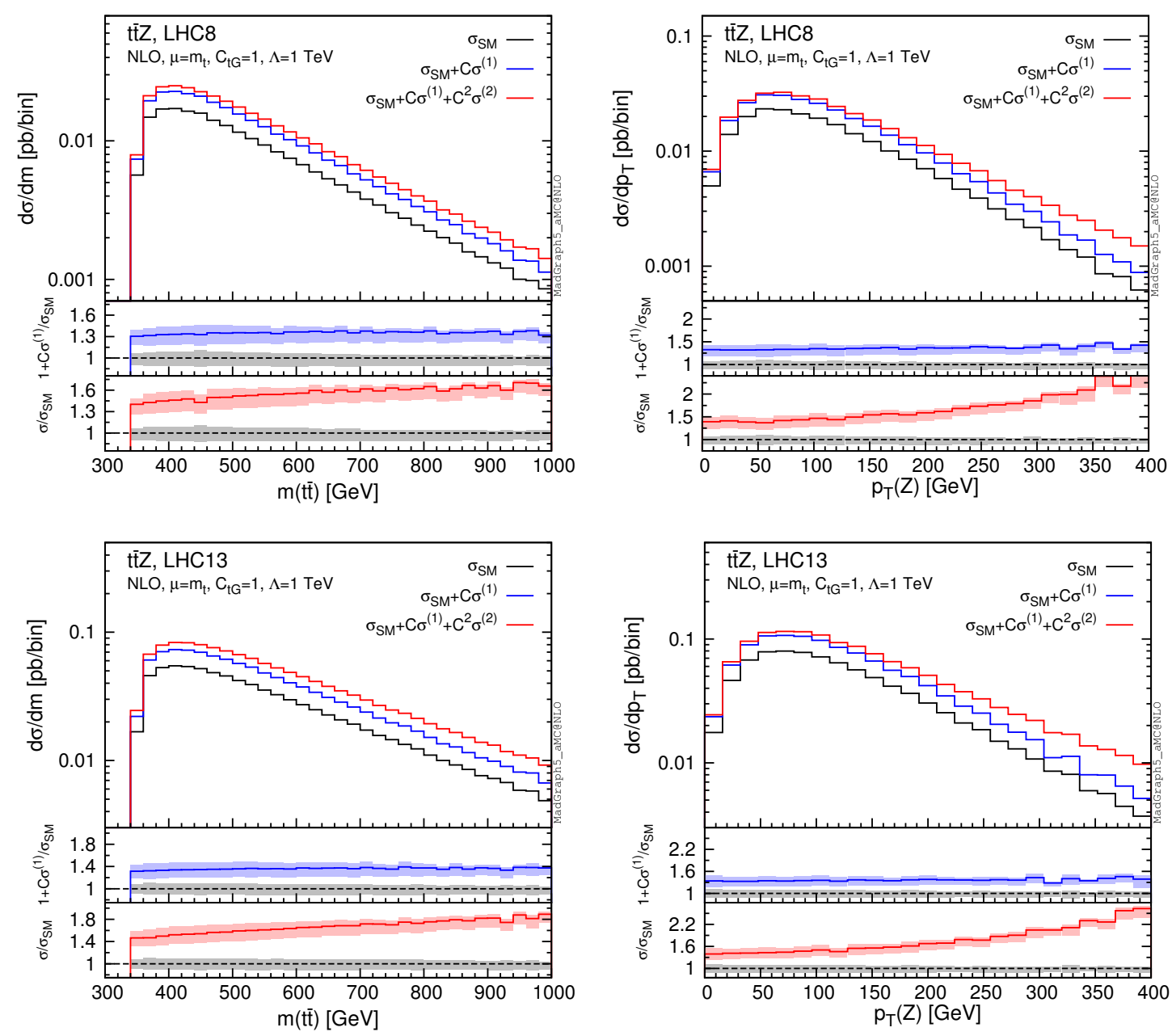

Figure 3. Invariant mass distributions for the top quark pair and $Z p_{T}$ distribution at 8 and $13 \mathrm{TeV}$ for the chromomagnetic operator for $C_{t G}=1$ and $\Lambda=1 \mathrm{TeV}$. Scale uncertainty bands are shown.

\subsection{Differential distributions}

Differential distributions are obtained at NLO for the $p p \rightarrow t \bar{t} Z, p p \rightarrow t \bar{t} \gamma$ and $p p \rightarrow$ $t \bar{t} \mu^{+} \mu^{-}$processes. This can be done also at NLO with matching to the PS, and with top quarks decayed while keeping spin correlations [50], all implemented in the MG5_AMC framework. Hence our approach can be used directly in realistic experimental simulation, with NLO+PS event generation, which allows for more detailed studies of possible EFT signals. In this work, for illustration purpose, we keep results simple by only presenting fixed order NLO distributions. No kinematical cuts are applied except for the $m(\ell \ell)>$ $10 \mathrm{GeV}$ and $p_{T}(\gamma)>20 \mathrm{GeV}$ generation cuts. We show results obtained with one non-zero operator coefficient at a time, with $\Lambda=1 \mathrm{TeV}$, and SM results are given for comparison.

We start by showing the distributions obtained for the $\mathcal{O}_{t G}$ operator at 8 and $13 \mathrm{TeV}$ in figure 3. We show as a reference the invariant mass distribution of the top quark pair and the transverse momentum of the $Z$. In the plots we show the SM prediction $\sigma_{\mathrm{SM}}$, 

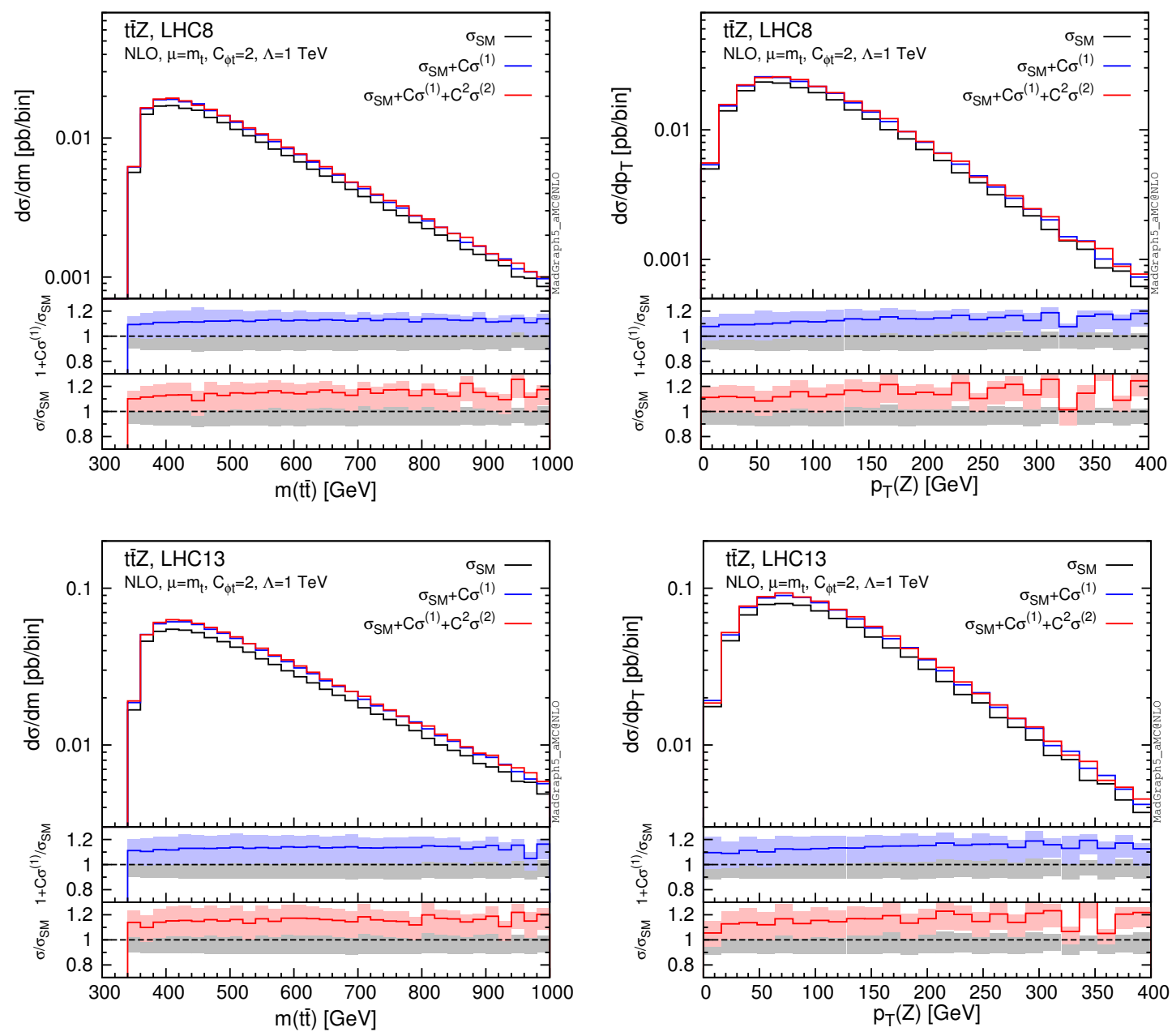

Figure 4. Invariant mass distributions for the top quark pair and $Z p_{T}$ distribution at 8 and $13 \mathrm{TeV}$ for the $\mathcal{O}_{\phi t}$ operator for $C_{\phi t}=2$ and $\Lambda=1 \mathrm{TeV}$. Scale uncertainty bands are shown.

the result for $C_{t G}=1, \Lambda=1 \mathrm{TeV}$ i) adding only the interference $\sigma_{i}^{(1)}$ and ii) adding both the interference and the squared terms $\sigma_{i i}^{(2)}$. We also include the corresponding ratios over the SM prediction and the scale uncertainty bands. It is clear that while the interference contribution is not changing the distribution shape, the $\mathcal{O}\left(\Lambda^{-4}\right)$ contribution is growing fast at high energies with the effect being more evident at $13 \mathrm{TeV}$ in both distributions shown here. Similar observations can be made for other observables, such as the transverse momentum of the top.

Results for the $\mathcal{O}_{\phi t}$ and $\mathcal{O}_{\phi Q}^{(1)}$ are shown in figure 4 and 5 respectively. In this case, we set the Wilson coefficients to 2 , in order to obtain visible deviations from the SM. These values are allowed by the current constraints. For these operators the $\mathcal{O}\left(\Lambda^{-4}\right)$ contribution is significantly smaller than the $\mathcal{O}\left(\Lambda^{-2}\right)$ and does not significantly alter the shape of the differential distributions as seen in the flat ratios for both the $t \bar{t}$ invariant mass and $Z p_{T}$ distributions. Results for $\mathcal{O}_{\phi Q}^{(3)}$ are identical to those of $\mathcal{O}_{\phi Q}^{(1)}$ (with a relative sign for $\sigma_{i}^{(1)}$ ), so we do not include them for brevity. 

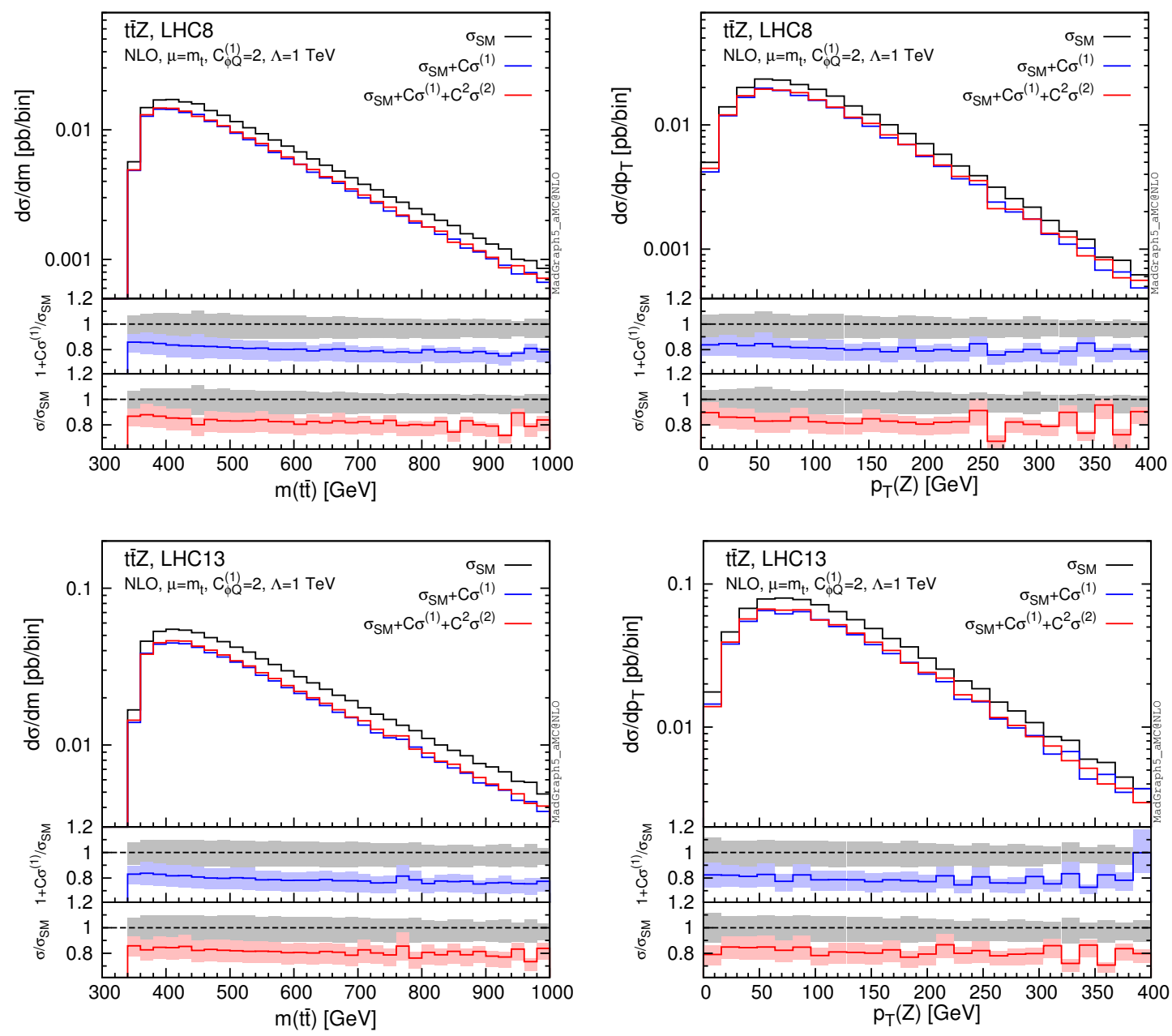

Figure 5. Invariant mass distributions for the top quark pair and $Z p_{T}$ distribution at 8 and $13 \mathrm{TeV}$ for the $\mathcal{O}_{\phi Q}^{(1)}$ operator for $C_{\phi Q}^{(1)}=2$ and $\Lambda=1 \mathrm{TeV}$. Scale uncertainty bands are shown.

For the $\mathcal{O}_{t W}$ and $\mathcal{O}_{t B}$ operators the EFT contributions are very small compared to the SM. In this case, we resort to $C_{t B}=4$ to demonstrate the effect of the $\mathcal{O}_{t B}$ operator in figure 6. For this operator the interference with the SM is much smaller than the $\mathcal{O}\left(\Lambda^{-4}\right)$ terms which are rising with the energy, as evident in the ratio plots.

For $t \bar{t} \gamma$, the results for $\mathcal{O}_{t G}$ operators are shown in figure 7 for 8 and $13 \mathrm{TeV}$. We notice that, in contrast with $t \bar{t} Z$, where the squared terms grow rapidly with the energy, that contribution is smaller for $t \bar{t} \gamma$ and does not lead to significant changes in the shapes of the two observables shown here. A comparison of the two processes can be made at the partonic cross-section level as shown in figure 8. In this plot the total cross-section is shown, i.e., schematically $\sigma_{S M}+C \sigma^{(1)}+C^{2} \sigma^{(2)}$ for the chromomagnetic operator. The $t \bar{t} Z$ cross-section is decomposed into the transverse and longitudinal $Z$ contributions. The only component that is rising with the energy is the longitudinal one, which explains why the photon distributions do not show any increase with the energy, while those for the $Z$ rise fast. In fact in $t \bar{t} Z$, the Higgs field in $\mathcal{O}_{t G}$ always takes its vacuum expectation value, and so by power counting the squared amplitude scales at most as $\sim s v^{2} / \Lambda^{4}$ for $t \bar{t} Z_{T}$ and 

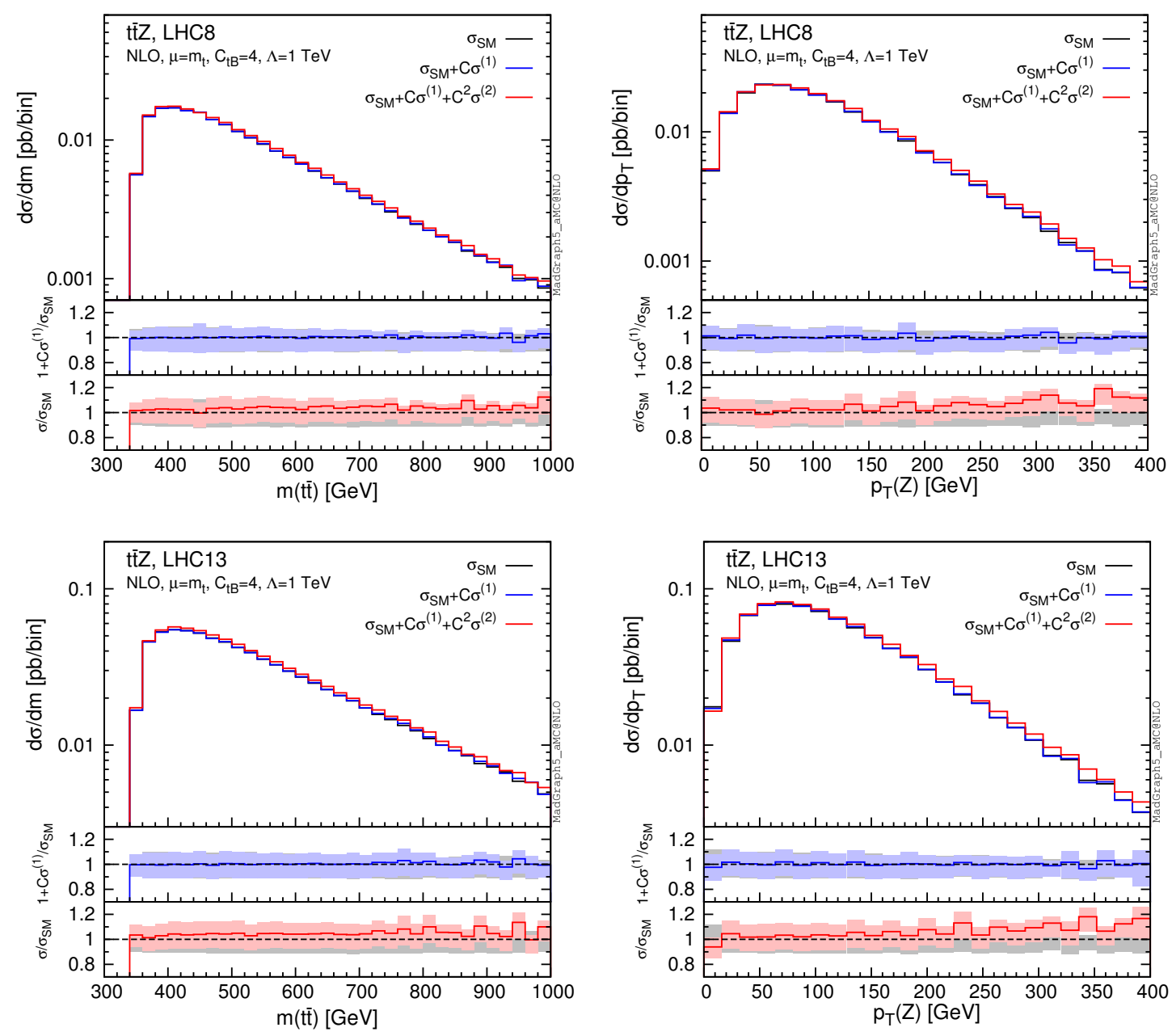

Figure 6. Invariant mass distributions for the top quark pair and $Z p_{T}$ distribution at 8 and $13 \mathrm{TeV}$ for the $\mathcal{O}_{t B}$ operator for $C_{t B}=4$ and $\Lambda=1 \mathrm{TeV}$. Scale uncertainty bands are shown.

$t \bar{t} \gamma$, which is not enough for the cross section to rise at high energy. On the other hand, in $t \bar{t} Z_{L}$ the longitudinal polarisation vector contributes an additional factor of $\left(E / m_{Z}\right)^{2}$, leading to a final $\sim s^{2} / \Lambda^{4}$ scaling of the squared amplitude. According to the Goldstoneboson equivalence theorem, the process $p p \rightarrow t \bar{t} G^{0}$, where $G^{0}$ is the neutral Goldstone boson, has the same energy dependence. This dependence comes solely from the diagram with a five-point contact interaction, $g g t t G^{0}$, from $\mathcal{O}_{t G}$, and because here the Higgs field is dynamical by simple power counting the square amplitude indeed scales as $\sim s^{2} / \Lambda^{4}$. To verify this reasoning, we have checked that in the process $g t \rightarrow t Z_{L}$, the squared amplitude rises as $\sim s^{2} / \Lambda^{4}$, and the leading term in the energy expansion can be fully reproduced by a single diagram with a contact $g t t G^{0}$ interaction. An analogous example of a high-energy growth is discussed in [51] where $t W \rightarrow t W$ scattering in $t \bar{t} W j$ electroweak production is employed to provide information on the top- $Z$ couplings.

The corresponding distributions for $\mathcal{O}_{t B}$ are shown in figure 9 for 8 and $13 \mathrm{TeV}$. As setting $C_{t B}=1$ does not give any visible deviations from the SM, we resort to $C_{t B}=4$ for these plots. While the squared term does not rise with $m(t \bar{t})$, it increases fast with 

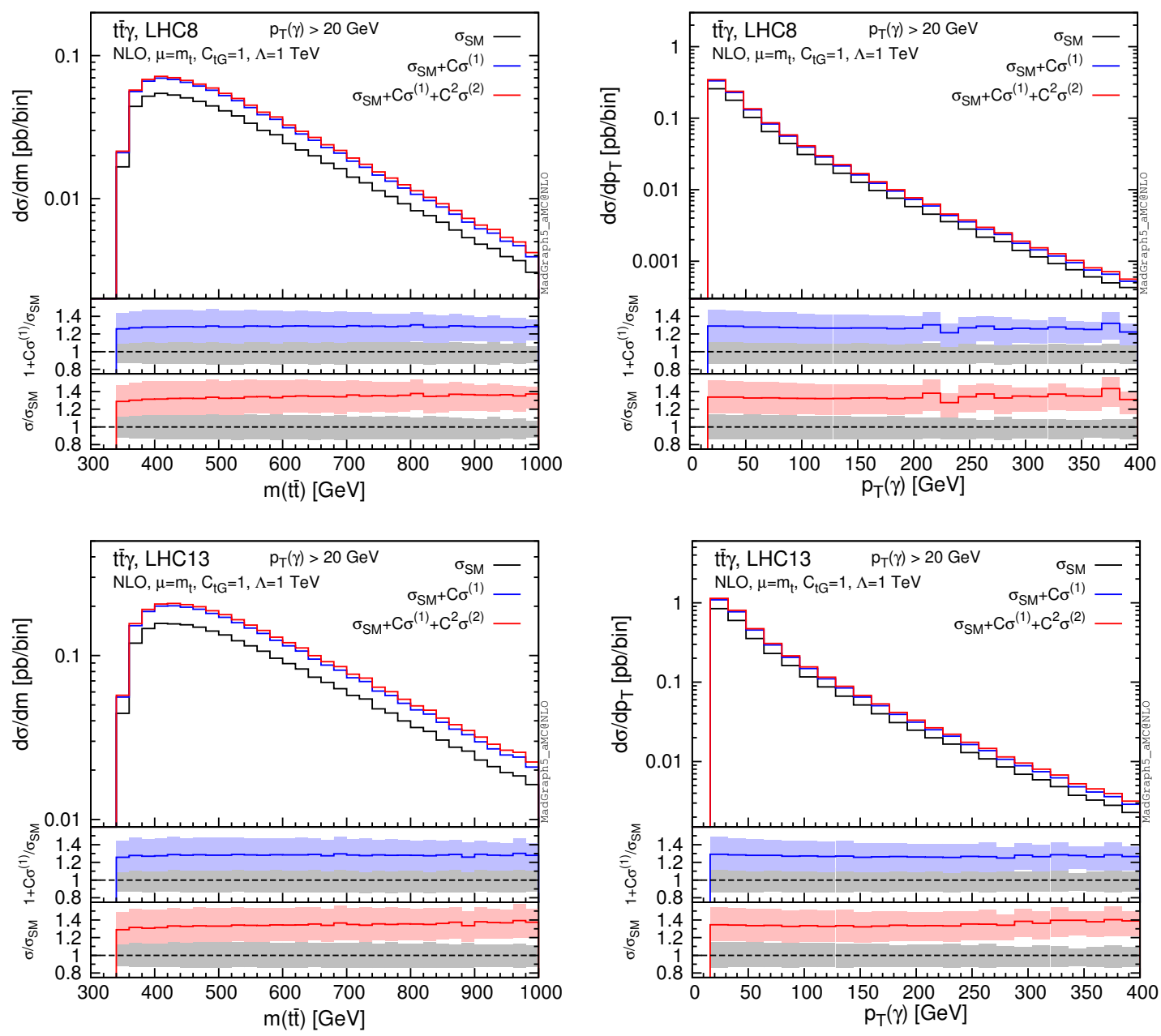

Figure 7. Invariant mass distributions for the top quark pair and photon $p_{T}$ distribution at 8 and $13 \mathrm{TeV}$ for the chromomagnetic operator for $C_{t G}=1$ and $\Lambda=1 \mathrm{TeV}$. Scale uncertainty bands are shown.

the photon transverse momentum. This is again related to the amount of momentum passing through the EFT vertex. High top pair invariant mass does not correspond to high momentum through the EFT vertex for the $\mathcal{O}_{t B}$ operator, in contrast with the situation for $\mathcal{O}_{t G}$. For $\mathcal{O}_{t G}$ there is a strong correlation between the $m(t \bar{t})$ and the energy in the EFT vertex leading to a rising distribution.

For the $t \bar{t} \mu^{+} \mu^{-}$process, we examine the angular separation between the leptons $\Delta \phi$ and the invariant mass distribution of the two leptons $m(\ell \ell)$ for the $\mathcal{O}_{t G}$ operator in figure 10 for 8 and $13 \mathrm{TeV}$. The angular separation between the two leptons is highly correlated with the transverse momentum of the vector boson. This implies that at low $\Delta \phi$, the behaviour matches that of the high vector transverse momentum region, since for a boosted vector boson, the leptons are collimated. As expected, the behaviour close to the $Z$ mass peak resembles that of the $t \bar{t} Z$ process, while at low invariant mass of the lepton pair it approaches that of $t \bar{t} \gamma$. 


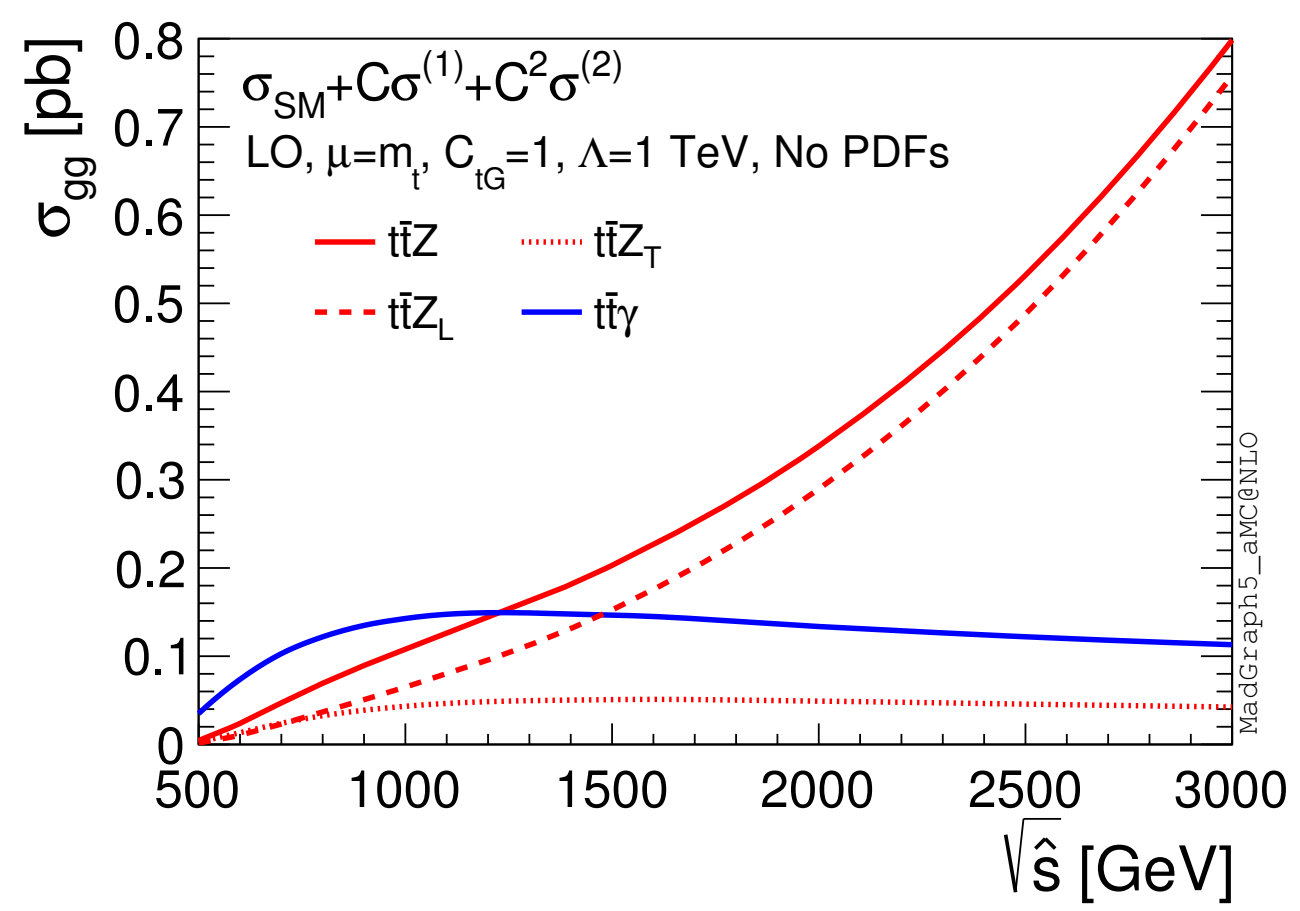

Figure 8. Partonic cross section for $t \bar{t} \gamma$ and $t \bar{t} Z$ as function of the centre of mass energy for the chromomagnetic operator. The $t \bar{t} Z$ cross section is decomposed to transverse and longitudinal $Z$ contributions.

The corresponding results for $\mathcal{O}_{\phi Q}^{(1)}$ are shown in figure 11. Again the behaviour of the ratios follows that of the $t \bar{t} Z$ close to the $Z$ mass peak, while at low masses the dimensionsix contribution approaches zero as this operator has no effect on the $t \bar{t} \gamma^{*}$ process which dominates at low $m(\ell \ell)$. The $\Delta \phi$ distributions are rather flat similarly to those of the $p_{T}(Z)$ for the same operator. For brevity we do not show the results for the rest of the current operators, as they are similar to $\mathcal{O}_{\phi Q}^{(1)}$.

We conclude our $t \bar{t} \mu^{+} \mu^{-}$discussion by showing the results for the $\mathcal{O}_{t B}$ operator operator in figure 12. The size of the interference with the SM increases at high lepton pair invariant masses while it is constant as a function of the angular separation between the leptons. The squared terms rise at high invariant mass and low angular separation in agreement with the observations made for the $t \bar{t} \gamma$ and $t \bar{t} Z$ distributions.

We conclude this section by commenting on the differential $K$-factors of the EFT contributions. As already mentioned in section 4.1, by comparing table 1 with tables $2-6$, one can see that the SM global $K$-factors are in general different from the $K$-factors of the EFT operators. This shows that already at the cross-section level, using the SM $K$-factor to estimate the NLO QCD corrections of the EFT contribution is not a reliable approximation. The same applies at the differential level. To demonstrate this observation, we present in figure 13 a comparison of the differential $K$-factors for the SM and EFT contributions. We focus on the $t \bar{t} \gamma$ and $t \bar{t} Z$ processes at $13 \mathrm{TeV}$ and show four representative observables. In 

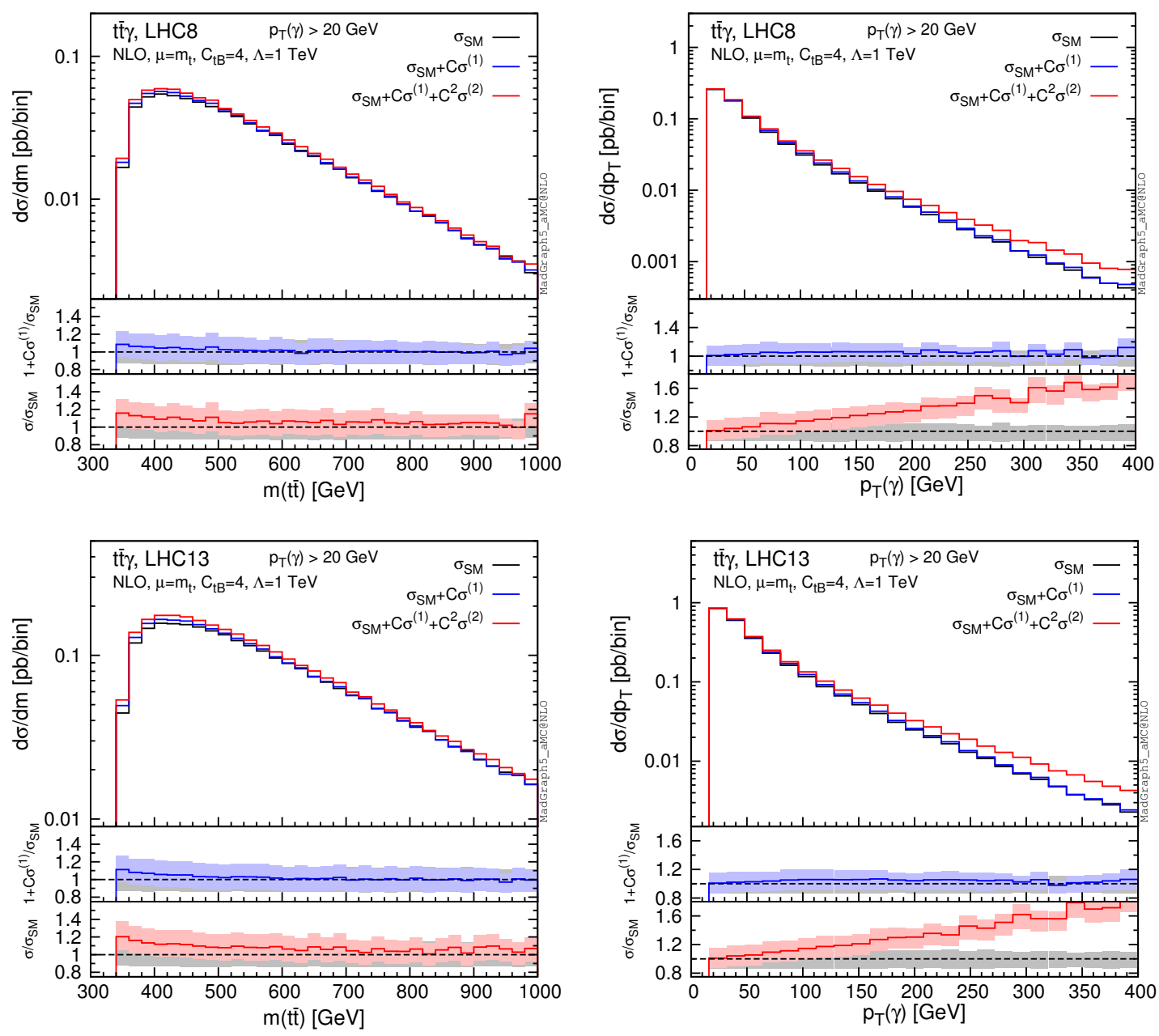

Figure 9. Invariant mass distributions for the top quark pair and photon $p_{T}$ distribution at 8 and $13 \mathrm{TeV}$ for the $\mathcal{O}_{t B}$ operator for $C_{t B}=4$ and $\Lambda=1 \mathrm{TeV}$. Scale uncertainty bands are shown.

the main panels we show the NLO and LO results of the $\sigma_{S M}$ and $\sigma_{S M}+C \sigma^{(1)}$. In the first two insets we present the differential $K$-factors of i) the SM $\left(K\left(\sigma_{S M}\right)\right)$ and ii) the operator contribution $\left(K\left(\sigma^{(1)}\right)\right)$, while in the third inset we show the ratio $(R)$ between the two. The comparison between table 1 and table 4 shows that the $O_{t B}$ global $K$-factor is lower than the SM one for the $t \bar{t} \gamma$ process. On top of that, in the top left plot of figure 13 we see that at differential level the ratio of $K$-factors is not flat. For the same process at the cross-section level the $O_{t G}$ contribution and the SM have similar $K$-factors. However, the top right plot reveals that the ratio $R$ is again not flat. Therefore even a bin-by-bin rescaling of the LO $O_{t G}$ distribution with the SM differential $K$-factor would lead to the mismatch depicted in the third inset. In the two lower plots we show results for the $t \bar{t} Z$ process for the $O_{t G}$ operator where similar observations can be made, highlighting the need for NLO QCD predictions for the EFT contributions. 

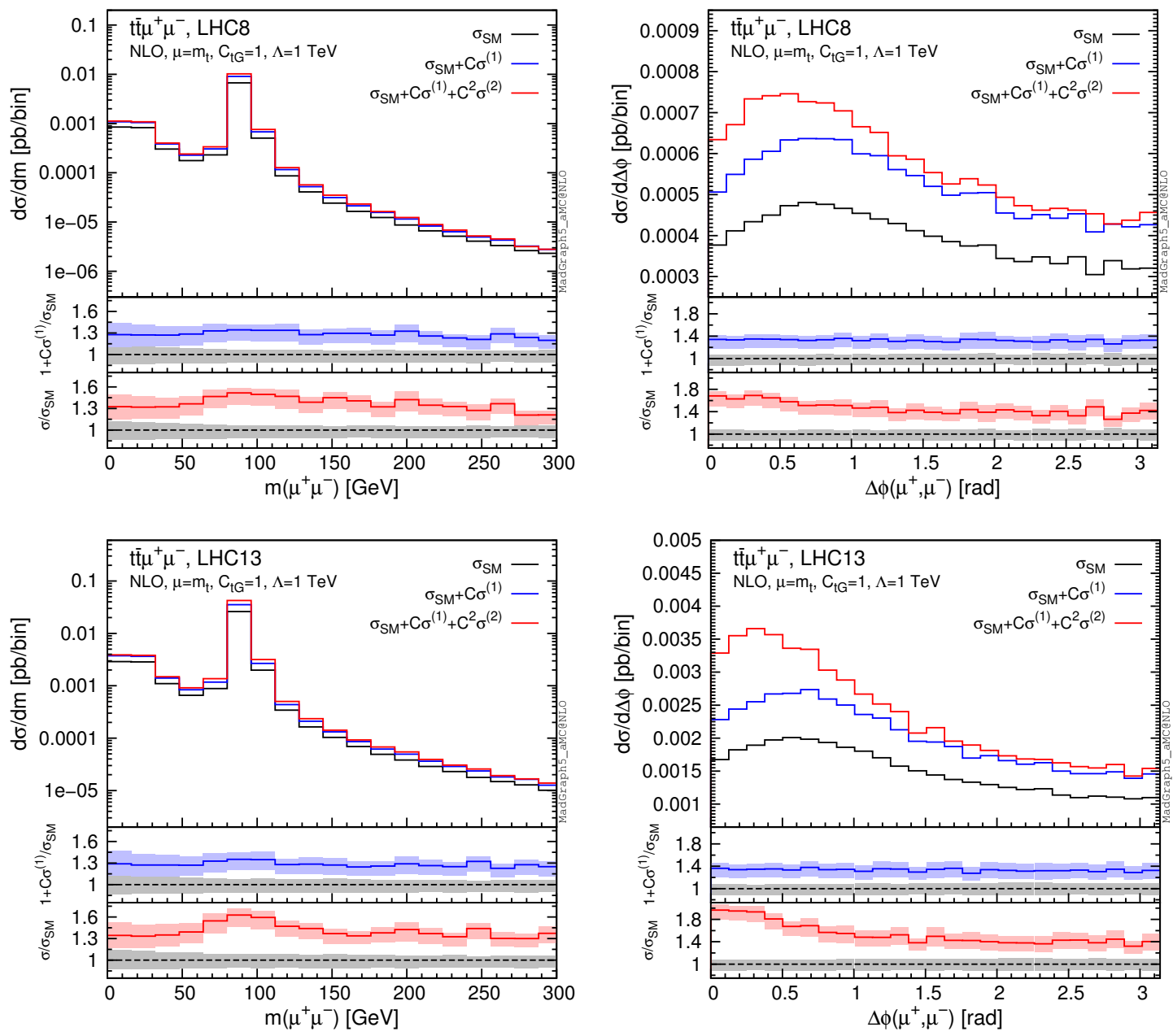

Figure 10. Invariant mass distributions for the lepton pair and lepton angular separation distribution at 8 and $13 \mathrm{TeV}$ for the chromomagnetic operator for the $\mathcal{O}_{t G}$ operator for $C_{t G}=1$ and $\Lambda=1 \mathrm{TeV}$.

\section{$5 \quad$ Results for $g g \rightarrow H Z$}

A subset of the operators affecting $t \bar{t} Z / t \bar{t} \gamma$ enter also in the associated production of a $H Z$ pair in gluon fusion, shown in the Feynman diagrams of figure 14. This process is formally part of the NNLO cross section for $H Z$ production and contributes at the $10 \%$ level. It is nevertheless particularly important in the high Higgs $p_{T}$ regions where the experimental searches are most sensitive. This process has been studied within the SM, also including the contribution of additional jet radiation, which turns out to be important in the high $p_{T}$ regions [52]. In this work, we consider this process as it can provide additional information on the Wilson coefficients once combined with the corresponding $H Z$ measurements at the LHC. In this section, we investigate the effect of the operators presented above on this process. We note that we consider only the operators involving the top quark and ignore all other dimension-six operators, such as those affecting the interaction of the Higgs with 

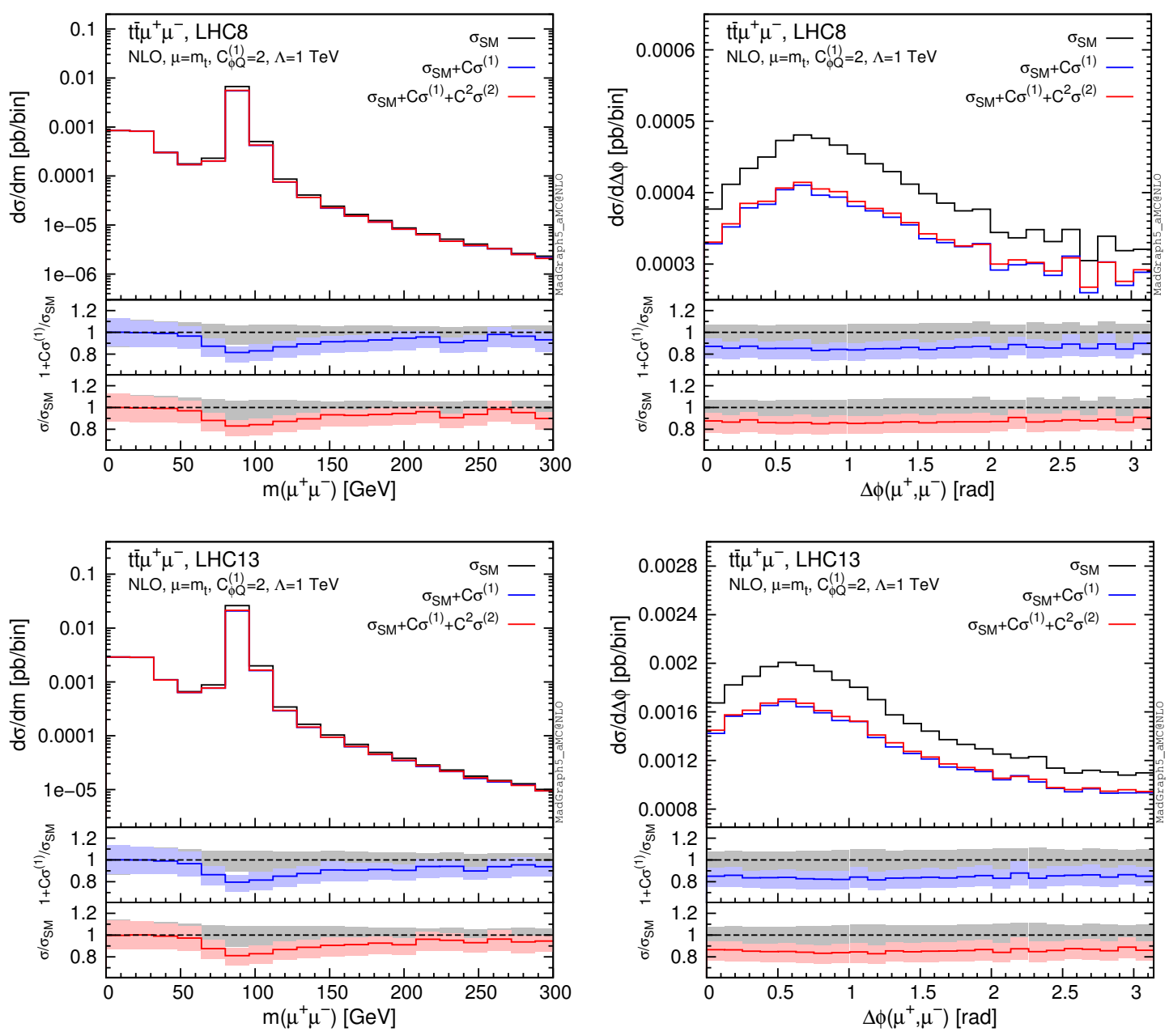

Figure 11. Invariant mass distributions for the lepton pair and lepton angular separation distribution at 8 and $13 \mathrm{TeV}$ for the $\mathcal{O}_{\phi Q}^{(1)}$ operator for $C_{\phi Q}^{(1)}=2$ and $\Lambda=1 \mathrm{TeV}$.

the vector bosons. In addition to modifying the interactions in the SM-like diagrams of figure 14, the dimension-six operators introduce additional vertices and hence Feynman diagrams as shown in figure 15.

For this process, the factorisation and renormalisation scale is set to $m_{H}=125 \mathrm{GeV}$. Only LO results can be obtained as the NLO computation requires 2-loop multi-scale Feynman integrals which are currently not available. The results are shown in table 7 for both the SM and the dimension-six operators cross sections, the corresponding scale uncertainties and the corresponding cross-section ratios for 8 and $13 \mathrm{TeV}$. The $\mathcal{O}_{t W}$ and $\mathcal{O}_{t B}$ operators do not contribute to this process, due to charge conjugation invariance. The $\mathcal{O}_{\phi Q}^{(3)}, \mathcal{O}_{\phi Q}^{(1)}$ and $\mathcal{O}_{\phi t}$ give the same contributions (with a relative minus sign as determined by eq. (A.3)) in the massless $b$-quark limit, as they affect in the same way the axial vector coupling of the top to the $Z$, which is the only component whose contribution is allowed by charge conjugation symmetry. If one wants to cancel the chiral anomaly in the triangle 

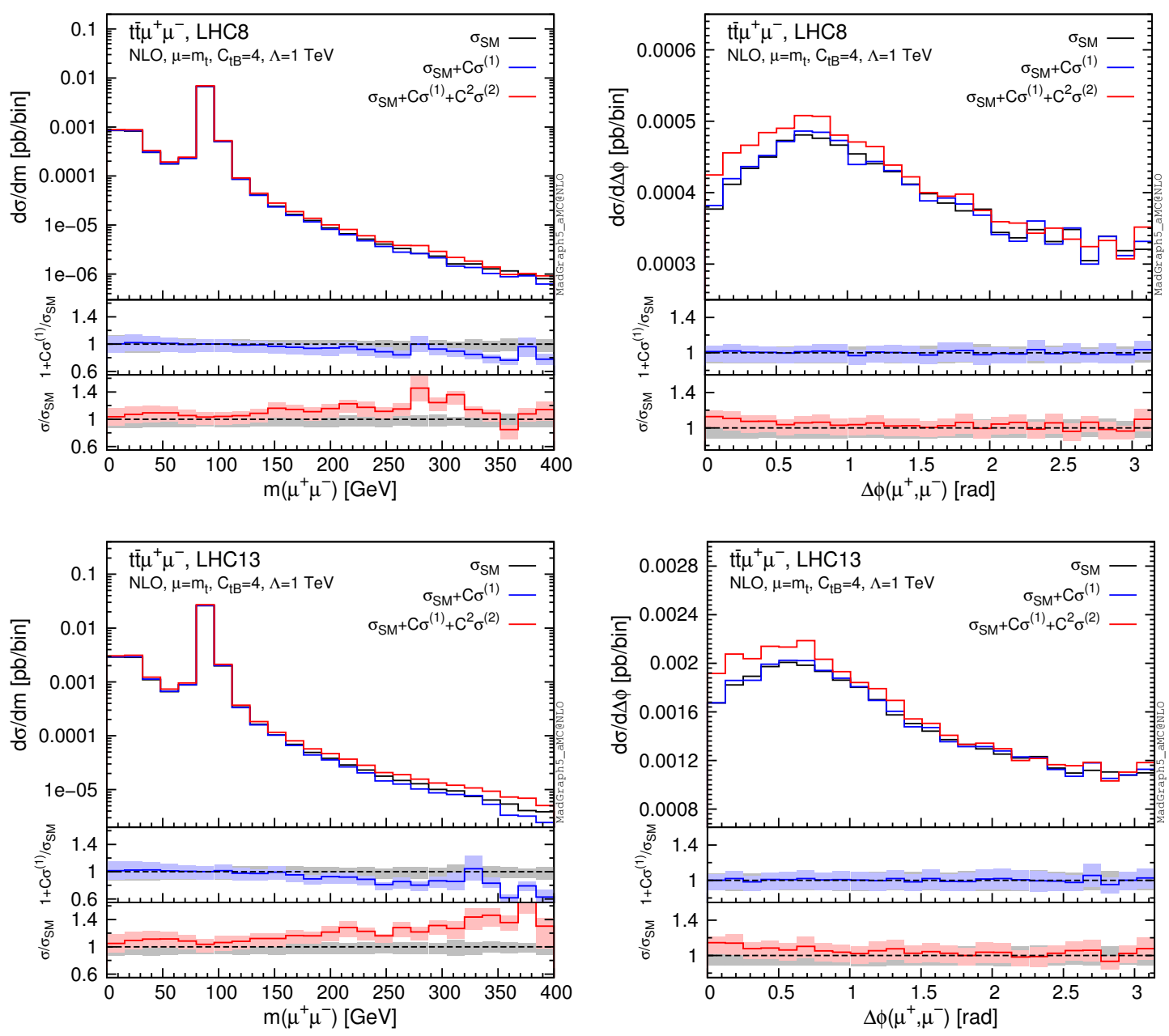

Figure 12. Invariant mass distributions for the lepton pair and lepton angular separation distribution at 8 and $13 \mathrm{TeV}$ for the $\mathcal{O}_{t B}$ operator for $C_{t B}=4$ and $\Lambda=1 \mathrm{TeV}$.

loop diagrams with the $Z$-boson in the $s$-channel, the $\mathcal{O}_{\phi b}$ operator can be included with its Wilson coefficient set to $C_{\phi b}=2 C_{\phi Q}^{(1)}-C_{\phi t}$. By appropriately fixing the coefficient of $\mathcal{O}_{\phi b}$, the axial-vector coupling of the bottom remains opposite to that of the top and the anomaly cancels. In practice this has a negligible numerical effect on the results.

The chromomagnetic operator gives a significant contribution reaching $35 \%$ of the SM cross section for $C_{t G}=1$ and $\Lambda=1 \mathrm{TeV}$. The three current operators give contributions at the $6 \%$ level. In both cases, the contribution of the squared amplitudes are subdominant at the total cross section level. These results suffer from large scale uncertainties as it is often the case with gluon fusion processes at LO. The invariant mass distribution for the $H Z$ pair is shown in figure 16 for the SM and the dimension-six operators. For this process, we find that both the interference with the SM amplitude and the squared contribution are growing with energy. 

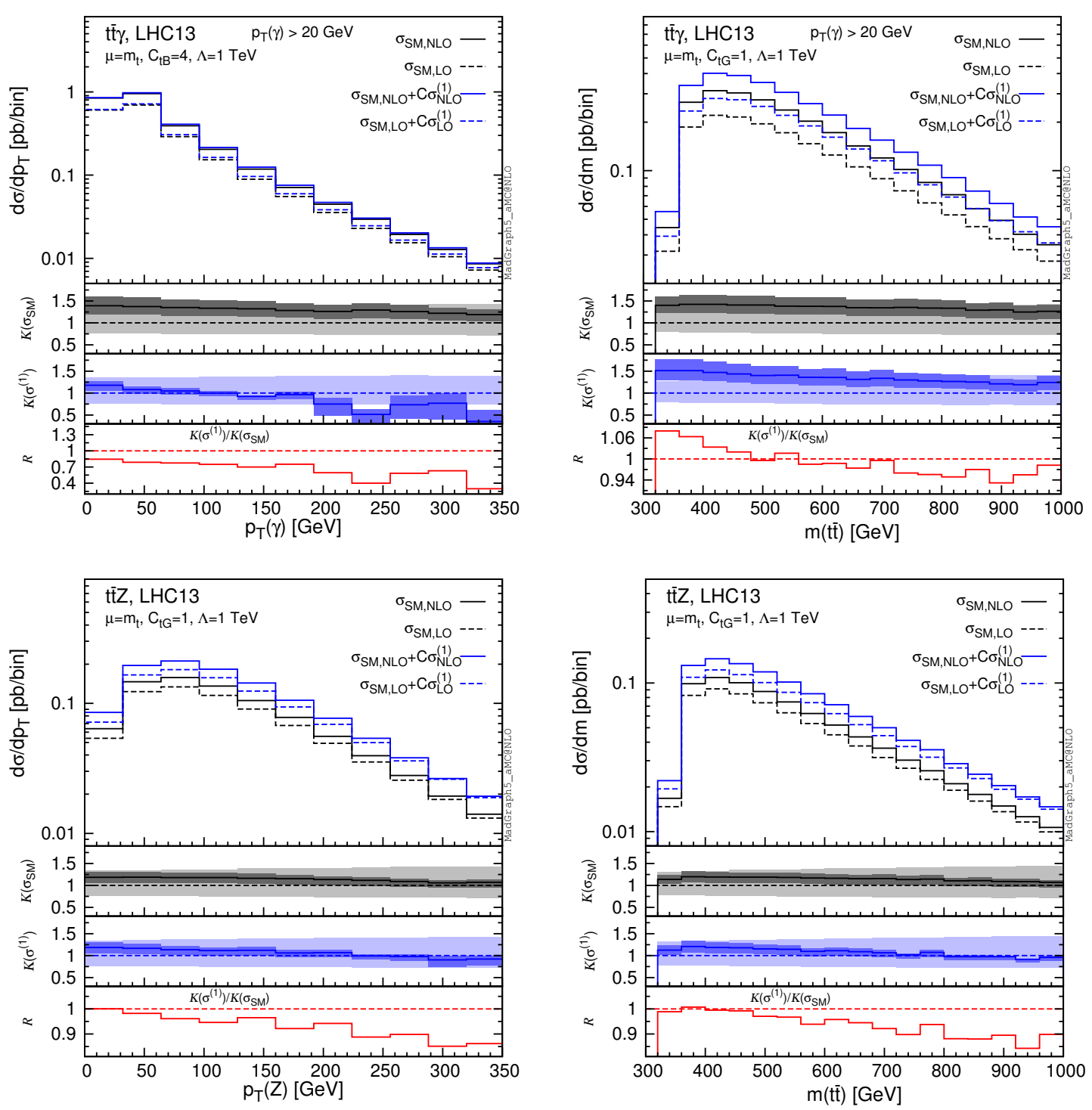

Figure 13. NLO and LO invariant mass of the top pair and $p_{T}$ of the vector boson distributions at $13 \mathrm{TeV}$ for $C_{t B}=4, C_{t G}=1$ and $\Lambda=1 \mathrm{TeV}$. Comparison between the SM and the interference term differential $K$-factors. Scale uncertainty bands are shown.

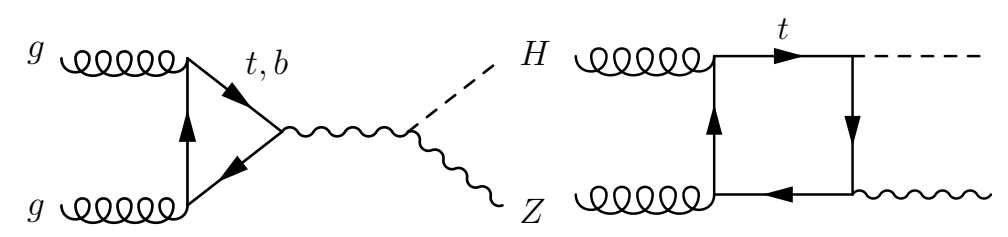

Figure 14. Feynman diagrams for $H Z$ production in gluon fusion in the SM. 

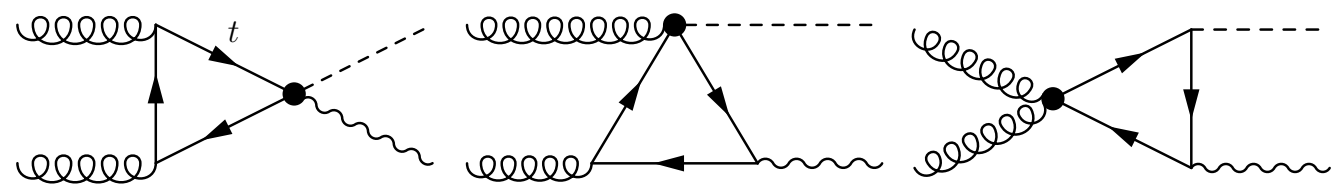

Figure 15. Additional types of Feynman diagrams for $H Z$ production in gluon fusion in the presence of dimension-six operators. The new vertices originating from the dimension-six operators are denoted with a blob.

\begin{tabular}{|c|c|ccc|}
\hline$[\mathrm{fb}]$ & $\mathrm{SM}$ & & $\mathcal{O}_{t G}$ & $\mathcal{O}_{\phi Q}^{(1)}$ \\
\hline & & $\sigma_{i}^{(1)}$ & $10.37_{-27.2 \%}^{+41.3 \%}$ & $1.719_{-27.6 \%}^{+42.5 \%}$ \\
& & $\sigma_{i i}^{(2)}$ & $1.621_{-28.7 \%}^{+45.1 \%}$ & $0.0469_{-29.2 \%}^{+46.5 \%}$ \\
& & & & \\
& & $\sigma_{i}^{(1)} / \sigma_{S M}$ & $0.356_{-0.8 \%}^{+0.9 \%}$ & $0.0590_{-1.4 \%}^{+1.8 \%}$ \\
& & $\sigma_{i i}^{(2)} / \sigma_{i}^{(1)}$ & $0.156_{-2.0 \%}^{+2.6 \%}$ & $0.0273_{-2.3 \%}^{+2.8 \%}$ \\
\hline \multirow{3}{*}{$13 \mathrm{TeV}$} & & $\sigma_{i}^{(1)}$ & $34.6_{-24.5 \%}^{+35.2 \%}$ & $5.91_{-24.9 \%}^{+36.4 \%}$ \\
& \multirow{3}{*}{$93.6_{-23.8 \%}^{+34.3 \%}$} & $\sigma_{i i}^{(2)}$ & $6.09_{-26.1 \%}^{+39.2 \%}$ & $0.182_{-26.6 \%}^{+40.2 \%}$ \\
& & $\sigma_{i}^{(1)} / \sigma_{S M}$ & $0.370_{-0.9 \%}^{+0.7 \%}$ & $0.0631_{-1.5 \%}^{+1.6 \%}$ \\
& & $\sigma_{i i}^{(2)} / \sigma_{i}^{(1)}$ & $0.176_{-2.1 \%}^{+2.9 \%}$ & $0.0309_{-2.2 \%}^{+2.8 \%}$ \\
\hline
\end{tabular}

Table 7. Cross sections (in fb) for $g g \rightarrow H Z$ production at the LHC at $\sqrt{s}=8 \mathrm{TeV}$ and $\sqrt{s}=$ $13 \mathrm{TeV}$ for the SM and the dimension-six operators. Scale uncertainties are shown in percentages.

\section{Results for the ILC}

The top-quark electroweak couplings can be accurately determined by future $e^{+} e^{-}$colliders, using top-pair production, thanks to the clean background. Our approach can be applied to $e^{+} e^{-}$colliders as well, providing more accurate predictions for deviations that will be measured in this process. In this section we present results obtained for the ILC at $\sqrt{s}=500 \mathrm{GeV}$ for top pair production. For this process, the $\mathcal{O}_{t G}$ operator contributes only at NLO, while the other operators contribute starting at LO. The results are presented in table 8. In this case, we do not show the renormalisation scale uncertainties as these can be computed only at NLO and are at the $1-2 \%$ level.

Unlike the $t \bar{t} V$ processes, here we find significant contributions from the dipole operators $\mathcal{O}_{t B}$ and $\mathcal{O}_{t W}$, while the other operators are suppressed, with $\mathcal{O}_{t G}, \mathcal{O}_{\phi Q}^{(1)}$ and $\mathcal{O}_{\phi Q}^{(3)}$ at the percent level, and $\mathcal{O}_{\phi t}$ at the per mille level. This is mainly because the momenta of $Z$ and $\gamma$ are at least at the $t \bar{t}$ threshold, and so the same dipole structure, which suppresses $t \bar{t} V$ production at the LHC, enhances the $t \bar{t}$ production at the ILC. It follows that the ILC could provide useful information complementary to the LHC as discussed also in $[18,19]$. We note here that the analysis of $[18,19]$ does not include the contribution from $\mathcal{O}_{t G}$, although (following an anomalous coupling approach) it does include the contribution of 

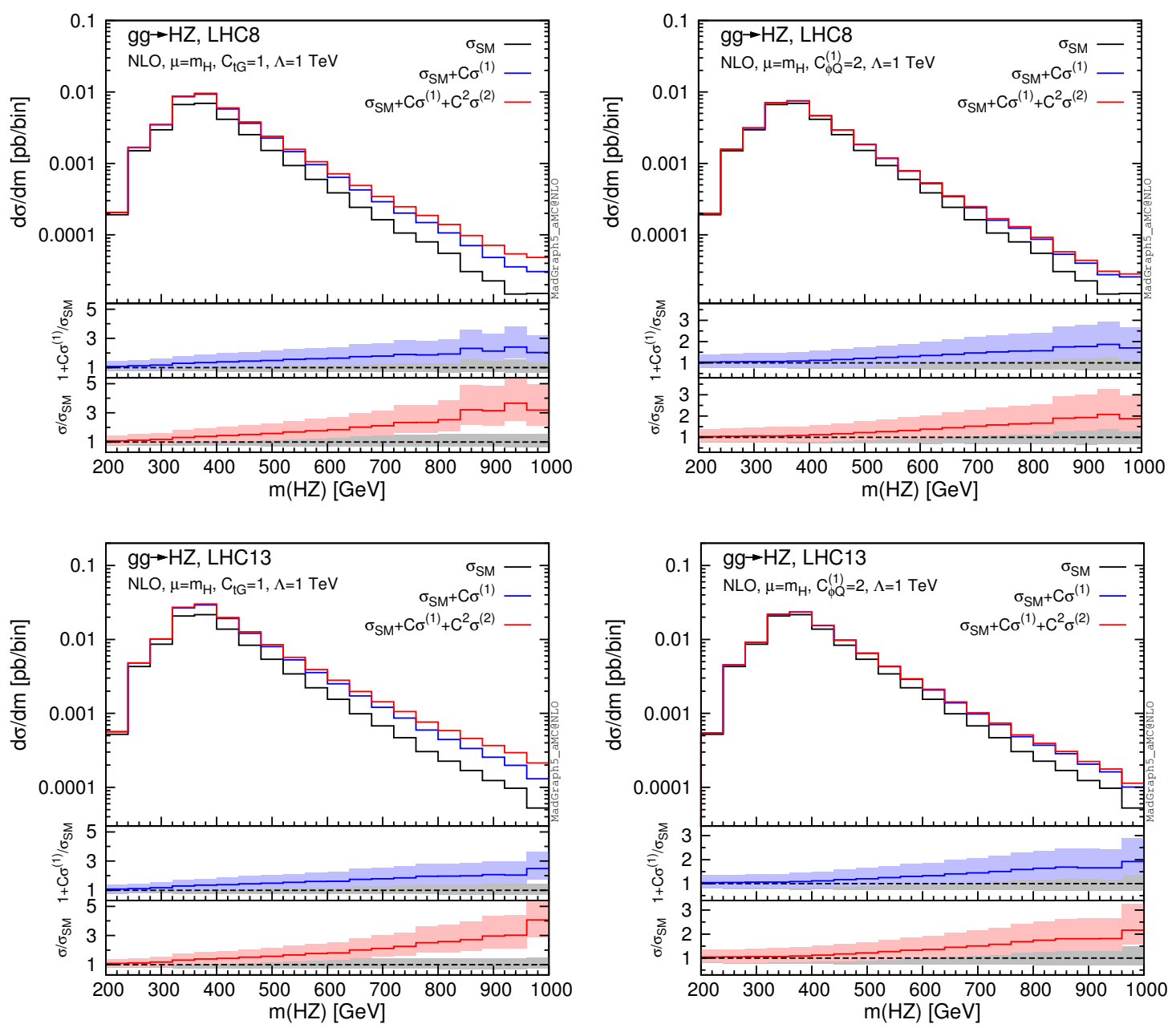

Figure 16. $H Z$ invariant mass distributions for $g g \rightarrow H Z$ at 8 and $13 \mathrm{TeV}$ for the $\mathcal{O}_{t G}$ and $\mathcal{O}_{\phi Q}^{(1)}$ operators. Scale uncertainty bands are shown.

the squares of the amplitudes with the top anomalous couplings and therefore also the CP-odd contributions.

\section{Theoretical uncertainties}

In this section we briefly discuss various theoretical uncertainties relevant to our results. In the SMEFT calculation there are two main types of theoretical uncertainties, those related to missing higher orders in the strong coupling and those from higher terms in the $1 / \Lambda$ expansion. In the former class, we can list

- Uncertainties due to parton-distribution functions.

This type of uncertainty is also present in the SM calculations and can be treated in the same way, i.e. by following the procedures associated with the corresponding 


\begin{tabular}{|cccccccc|}
\hline $500 \mathrm{GeV}$ & $\mathrm{SM}$ & $\mathcal{O}_{t G}$ & $\mathcal{O}_{\phi Q}^{(3)}$ & $\mathcal{O}_{\phi Q}^{(1)}$ & $\mathcal{O}_{\phi t}$ & $\mathcal{O}_{t W}$ & $\mathcal{O}_{t B}$ \\
\hline$\sigma_{i, L O}^{(1)}$ & 566 & 0 & 15.3 & -15.3 & -1.3 & 272 & 191 \\
$\sigma_{i, N L O}^{(1)}$ & 647 & -6.22 & 18.0 & -18.0 & -1.0 & 307 & 216 \\
$K$-factor & 1.14 & $\mathrm{~N} / \mathrm{A}$ & 1.17 & 1.17 & 0.78 & 1.13 & 1.13 \\
$\sigma_{i, L O}^{(2)}$ & & 0 & 0.72 & 0.71 & 0.72 & 60.4 & 27.2 \\
$\sigma_{i, N L O}^{(2)}$ & & 0.037 & 0.83 & 0.82 & 0.82 & 68.8 & 31.0 \\
$\sigma_{i, L O}^{(1)} / \sigma_{S M, L O}$ & & 0 & 0.027 & -0.027 & -0.0022 & 0.48 & 0.34 \\
$\sigma_{i, N L O}^{(1)} / \sigma_{S M, N L O}$ & & -0.096 & 0.028 & -0.028 & -0.0015 & 0.47 & 0.33 \\
$\sigma_{i, L O}^{(2)} / \sigma_{i, L O}^{(1)}$ & & $\mathrm{N} / \mathrm{A}$ & 0.047 & -0.047 & -0.57 & 0.22 & 0.14 \\
$\sigma_{i, N L O}^{(2)} / \sigma_{i, N L O}^{(1)}$ & & -0.006 & 0.046 & -0.046 & -0.82 & 0.22 & 0.14 \\
\hline
\end{tabular}

Table 8. Cross sections (in fb) for $t \bar{t}$ production at the ILC at $\sqrt{s}=500 \mathrm{GeV}$. Renormalisation scale uncertainties are not shown. They are only present at NLO and remain at the $1 \%$ level.

PDF sets, as long as the scale of new physics is high enough and the EFT operators do not modify the DGLAP equations.

- Uncertainties due to missing higher orders in the $\alpha_{s}$ expansion as in the SM.

This kind of uncertainty is typically estimated by varying the renormalisation and factorisation scales as done in SM calculations. All results presented in this work are provided along with uncertainties that are estimated by varying these two scales independently.

- Uncertainties due to missing higher orders in the $\alpha_{s}$ expansion of the EFT operators.

In the SMEFT an additional uncertainty, related to the scale at which the operators are defined, should be considered as well. It characterises the uncancelled logarithmic terms in the renormalisation group running and mixing of the operators. We did not evaluate these uncertainties explicitly even though it is possible in our framework. For the operators we have studied in this work, they are expected to be negligible compared to the first two scale uncertainties [17]. This is because the anomalous dimensions of the relevant operators happen to be smaller by roughly an order of magnitude compared to the beta function of $\alpha_{s}$ (see ref. [17] for a discussion of the operator scale uncertainty in the single-top processes).

We now consider uncertainties due to missing $\mathcal{O}\left(\Lambda^{-4}\right)$ contributions, also discussed in [53]. Up to this order, the cross section (or any other observable) can be written as:

$$
\sigma=\sigma_{S M}+\sum_{i} \frac{C_{i}^{\operatorname{dim} 6}}{(\Lambda / 1 \mathrm{TeV})^{2}} \sigma_{i}^{(1, \operatorname{dim} 6)}+\sum_{i<j} \frac{C_{i}^{\operatorname{dim} 6} C_{j}^{\operatorname{dim} 6}}{(\Lambda / 1 \mathrm{TeV})^{4}} \sigma_{i j}^{(2, \operatorname{dim} 6)}+\sum_{i} \frac{C_{i}^{\operatorname{dim} 8}}{(\Lambda / 1 \mathrm{TeV})^{4}} \sigma_{i}^{(1, \operatorname{dim} 8)}
$$


The last two terms are formally $\mathcal{O}\left(\Lambda^{-4}\right)$ contributions, and could in principle be neglected as they are expected to be suppressed for $\mathcal{O}(1)$ coefficients. One should then consider

- Impact of the squared contributions $\sigma_{i j}^{(2, \operatorname{dim} 6)}$ coming from dimension-six operators.

These contributions can be explicitly calculated with our approach, even though obtaining the complete results can be time consuming. In this work, we have always provided the results for $\sigma_{i i}^{(2)}$ for each operator $\mathcal{O}_{i}$, for not only total cross sections but also for distributions. In fact, one could include these squared contributions in the central values as part of the theoretical predictions, if only one operator is taken to be non-zero at a time. As we have mentioned, this can be justified for cases where the expansion in $E^{2} / \Lambda^{2}$ is under control but the squared contribution may still be large, due to less constrained operator coefficients, i.e. if $C_{i}^{2} \frac{E^{4}}{\Lambda^{4}}>C_{i} \frac{E^{2}}{\Lambda^{2}}>1>\frac{E^{2}}{\Lambda^{2}}$ is satisfied. In any case, our results for the $\sigma_{i i}^{(2)}$ terms can provide useful information for the evaluation of the uncertainties, if the squared contributions are neglected or only partly included.

As we have discussed already, the relative size of $\sigma_{i i}^{(2)}$ compared to $\sigma_{i}^{(1)}$ does not imply anything about the validity of the EFT and careful assessment should be done on a case-by-case basis.

- Validity of the EFT, i.e. contributions from missing higher-dimensional operators.

The second contribution at $\mathcal{O}\left(\Lambda^{-4}\right), \sigma_{i}^{(1, \operatorname{dim} 8)}$, comes from interference between $\mathrm{SM}$ and dimension-eight operators. These contributions cannot be computed in our approach, and will have to be neglected. A corresponding uncertainty should be taken into account. This can be easily done at the LO by calculating the interference contribution from typical dimension-eight operators. Alternatively, by simple power counting, these uncertainties may be estimated to be of order $C_{i}^{\operatorname{dim} 6} /(\Lambda / 1 \mathrm{TeV})^{2} \sigma_{i}^{(1, \operatorname{dim} 6)} s / \Lambda^{2}$. In this work, we do not assume a specific value of $\Lambda$, and so evaluating such uncertainties is not possible without additional assumptions. However, in a real analysis, for any given $\Lambda$, one can always apply a cut $s_{\max }$ on the centre-of-mass energy of the process, so that this uncertainty remains under control.

\section{Discussion}

In this section we explore the sensitivity of the top processes discussed above to the various operators. Experimental results from $[2,5,6,54]$ are used. For the $t \bar{t} Z$ measurement by ATLAS [6] and the $t \bar{t} \gamma$ measurement by CMS [2], a direct comparison is difficult, because of the way in which the measured cross sections are defined. We thus define the " $R$ " ratios in order to facilitate a direct comparison between the quoted experimental measurements and our theory predictions, as explained in appendix B. These ratios are always taken into account when experimental results on $t \bar{t} \mu^{+} \mu^{-}$and $t \bar{t} \gamma$ are used. On the other hand, the other measurements can be directly compared with our predictions.

We first examine the $\mathcal{O}_{t G}$ operator, which affects all production of top quark pairs with a vector boson, as well as $t \bar{t}$ production. The sensitivity of various processes to the 


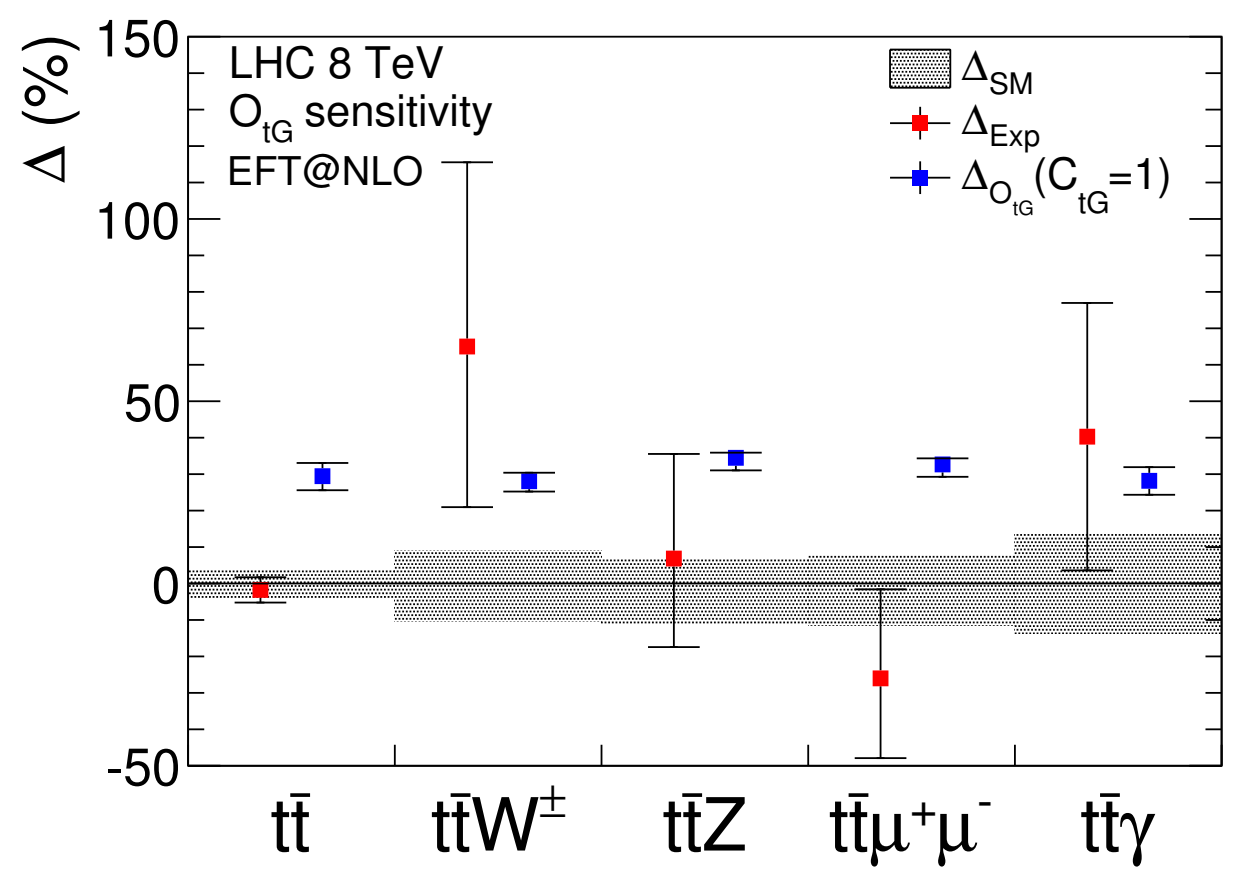

Figure 17. Sensitivity of various processes to the $\mathcal{O}_{t G}$ operator. $\Delta$ denotes the percentage difference from the SM theoretical prediction for each process. Theory predictions for all $t \bar{t} V$ processes are at NLO in QCD while for $t \bar{t}$ the NNLO result of [55] is employed. Experimental measurements are also shown along with the corresponding experimental uncertainties taken from [54] for $t \bar{t}$, [5] for $t \bar{t} W$ and $t \bar{t} Z,[6]$ for $t \bar{t} \mu^{+} \mu^{-}$and [2] for $t \bar{t} \gamma$.

$\mathcal{O}_{t G}$ operator is demonstrated in figure 17. In the plot we include the percentage deviation from the SM predictions for top pair production, and top pair production in association with a $W, Z$ boson or a photon, as well as the $t \bar{t} \ell^{+} \ell^{-}$process for $C_{t G}=1$ and $\Lambda=1 \mathrm{TeV}$. All SM predictions and uncertainties are given at NLO, apart from the top pair production cross-section, which is given at NNLO+NNLL [55]. We also present the experimental measurements and the corresponding uncertainties (systematic and statistical uncertainties added in quadrature). Only the $\mathcal{O}\left(1 / \Lambda^{2}\right)$ contribution is included. The $\mathcal{O}_{t G}$ operator affects all processes considered here in a similar way, at the $30 \%$ level for $\Lambda=1 \mathrm{TeV}$ and $C_{t G}=1$. At present, the most stringent direct constraints on this operator are obtained from the top pair production measurement, which is by far the most accurate one.

The relative sensitivity of the top processes to all operators can be summarised in figure 18, where the results for $C=1$ are shown as a ratio over the SM NLO cross sections, for the 6 operators considered here both at LO and NLO, along with the corresponding $K$-factors in the lower panel. The reduction of the theoretical uncertainties at NLO is also evident in the plot. The corresponding sensitivity plot for $13 \mathrm{TeV}$ is shown in figure 19, in which similar observations can be made.

Using the experimental measurements, one can further explore the sensitivity of the $t \bar{t} \gamma$ and $t \bar{t} Z$ processes on the various operators as shown in figures 20,21 and 22 . In the contour 


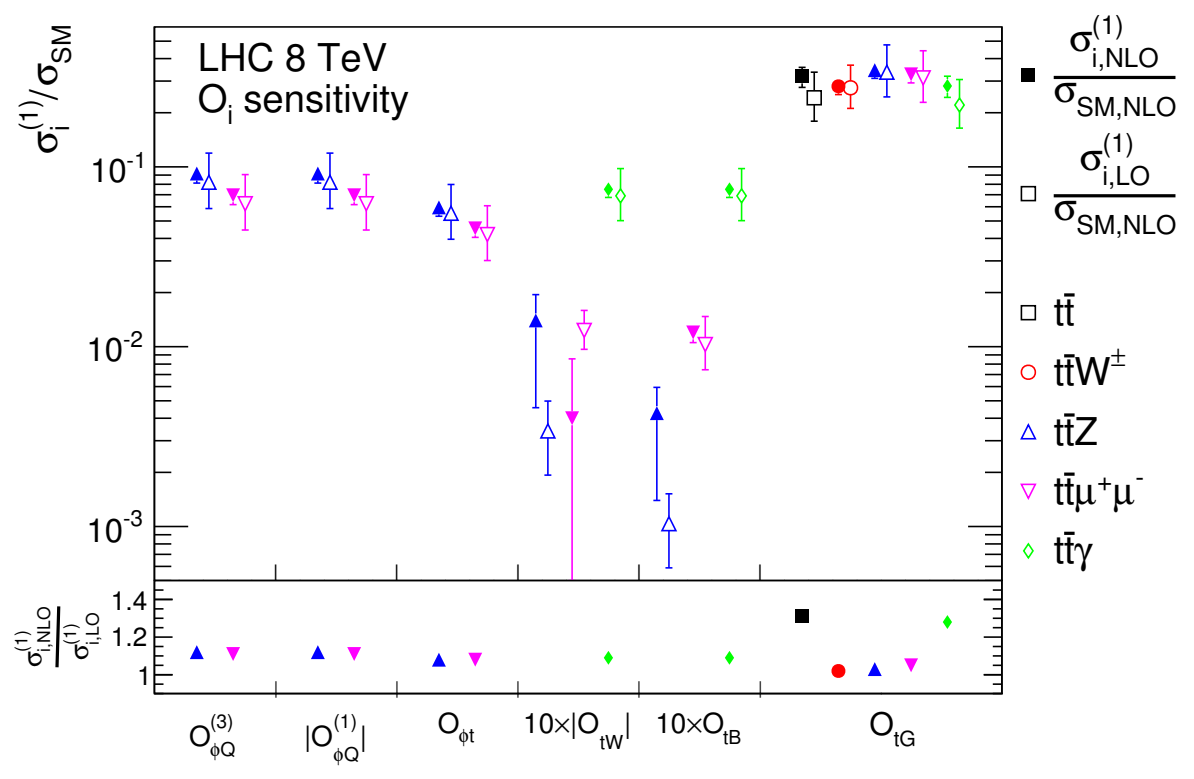

Figure 18. Sensitivity of various top quark processes to the various operators shown at LO and $\mathrm{NLO}$ at $8 \mathrm{TeV} . K$-factors are also shown for $\sigma_{i}^{(1)}$ as well as the scale uncertainties. We do not show the $K$-factors for the $\mathcal{O}_{t B}$ and $\mathcal{O}_{t W}$ operators in the $t \bar{t} Z$ and $t \bar{t} \mu^{+} \mu^{-}$processes, as in this case accidental cancellations lead to large or even negative $K$-factors.

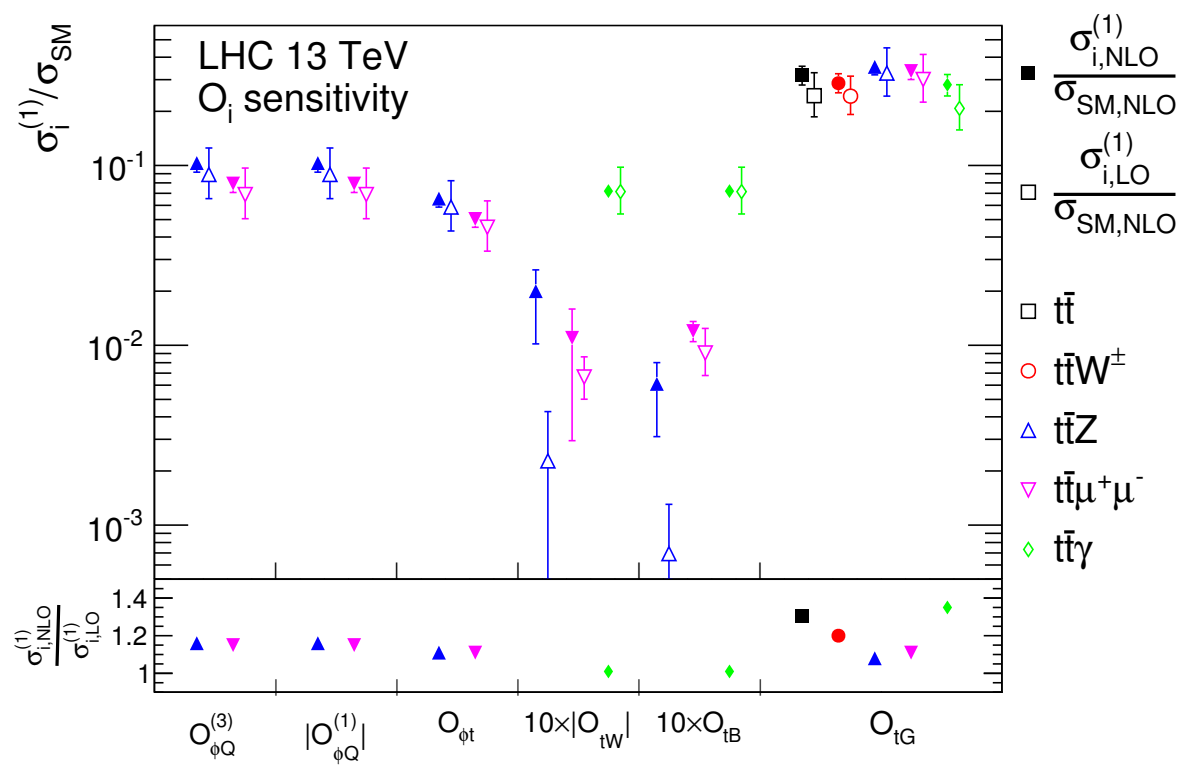

Figure 19. Sensitivity of various top quark processes to the various operators shown at LO and NLO at $13 \mathrm{TeV}$. Details as in figure 18. 


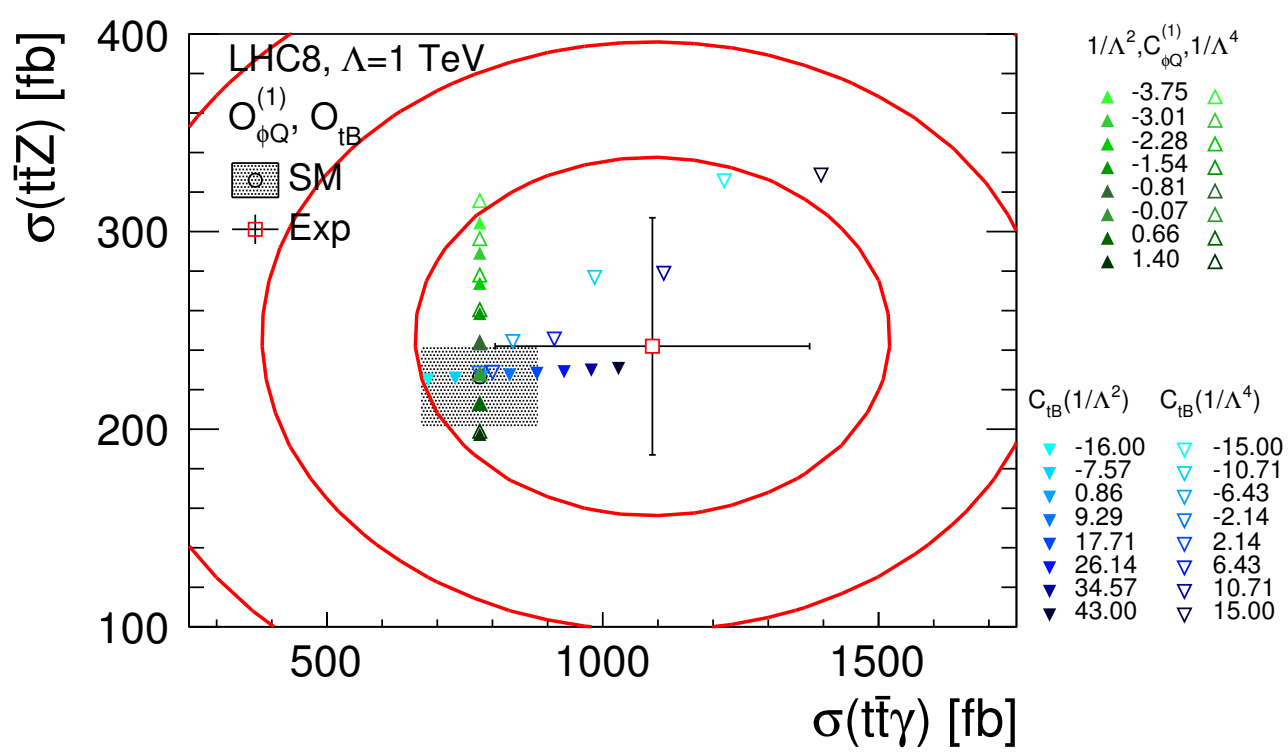

Figure 20. Sensitivity of the $t \bar{t} \gamma$ and $t \bar{t} Z$ processes to the $\mathcal{O}_{\phi Q}^{(1)}$ and $\mathcal{O}_{t B}$ operators. For each value of the coefficient we show the cross-section including i) only the interference term (filled triangles) and ii) both the interference and the squared contribution (unfilled triangles). The range for the Wilson coefficients is determined by the current constraints as discussed in section 2. The experimental measurements used in this plot are taken from [2] and [5] for $t \bar{t} \gamma$ and $t \bar{t} Z$ respectively. The squared contribution of the $\mathcal{O}_{t B}$ operator is very large, and therefore we employ a separate smaller interval to obtain cross sections within the boundaries of this plot.

plots we include the experimental results of [2] for $t \bar{t} \gamma$ and [5] for $t \bar{t} Z$ and the corresponding one and two sigma contour plots. In this case, we assume there is no correlation between the two measurements. The SM NLO predictions and the corresponding scale uncertainties are also shown in the plots. We plot the cross section obtained by varying the Wilson coefficients of the various operators. For clarity and to avoid overcrowding the contour plots, we present the operators in pairs. For the coefficients, we employ the current constraints to define our interval. Vertical lines in the plots indicate that the $t \bar{t} \gamma$ process is not affected by the specific operator, i.e. $\mathcal{O}_{\phi t}, \mathcal{O}_{\phi Q}^{(3)}$ and $\mathcal{O}_{\phi Q}^{(1)}$. Cross sections with and without adding the $\mathcal{O}\left(1 / \Lambda^{4}\right)$ contributions from the squared EFT amplitudes are compared. The $\mathcal{O}_{t B}$ operator is very loosely constrained, and therefore including the squared term for the large allowed values of the Wilson coefficient has an enormous effect on the cross sections, as the $\mathcal{O}\left(1 / \Lambda^{4}\right)$ contribution scales like $C_{t B}^{2}$. For the more constrained current operators $\mathcal{O}_{\phi Q}^{(1)}$ and $\mathcal{O}_{\phi Q}^{(3)}$, the squared contribution becomes important only at the edges of the allowed intervals. We also notice that for the $\mathcal{O}_{\phi t}$ and $\mathcal{O}_{t G}$ operators the $\mathcal{O}\left(1 / \Lambda^{4}\right)$ contribution is important for a sizeable part of allowed interval, in the first case because the constraints are rather loose and in the second case because $\sigma_{t G}^{(2)}$ is large. Finally, we note that the contour plots qualitatively demonstrate the size of the experimental uncertainties needed for these processes to have an impact on the allowed values of the coefficients. In that respect we observe for example that the $\mathcal{O}_{t W}$ operator receives very stringent constraints 


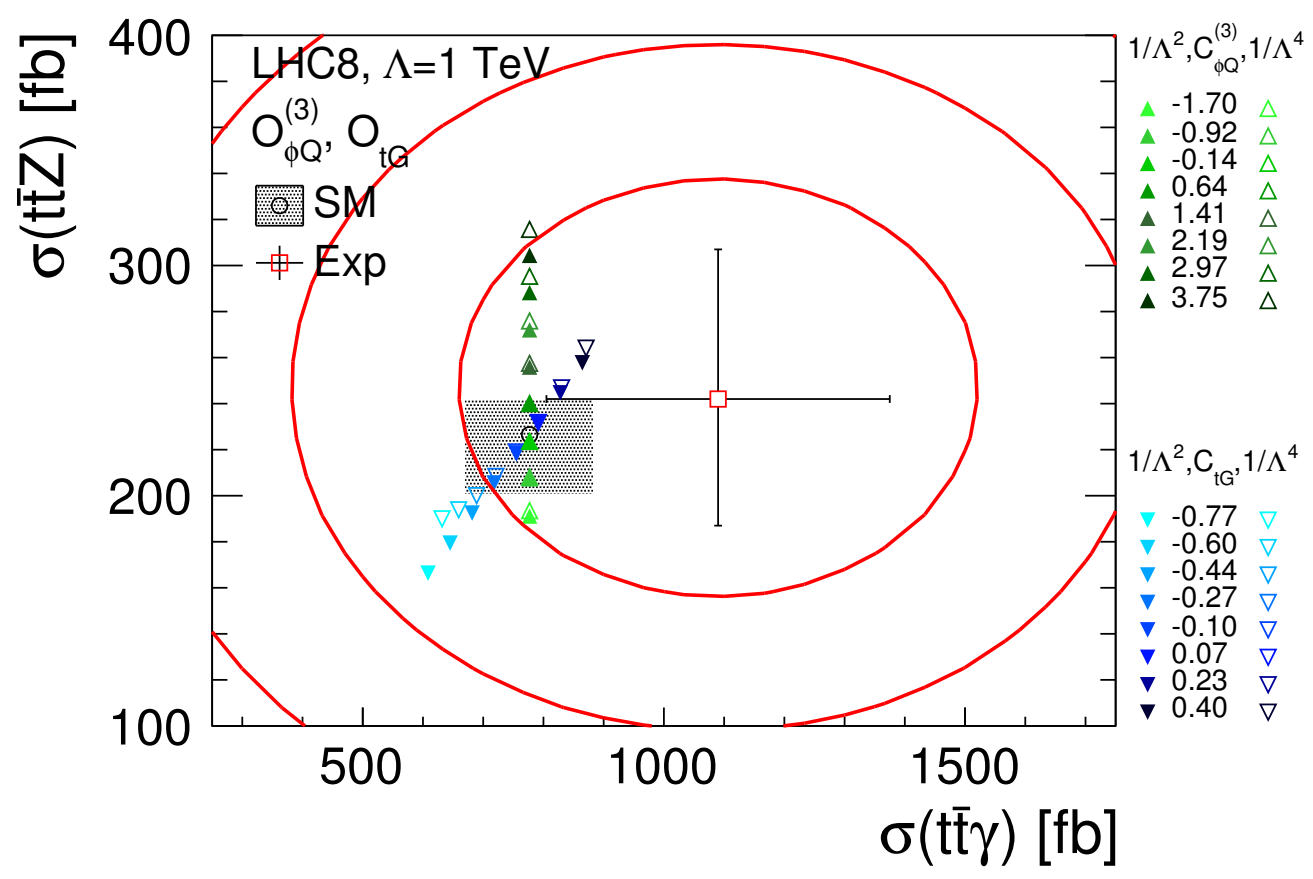

Figure 21. Sensitivity of the $t \bar{t} \gamma$ and $t \bar{t} Z$ processes to the $\mathcal{O}_{\phi Q}^{(3)}$ and $\mathcal{O}_{t G}$ operators. Details as in figure 20 .

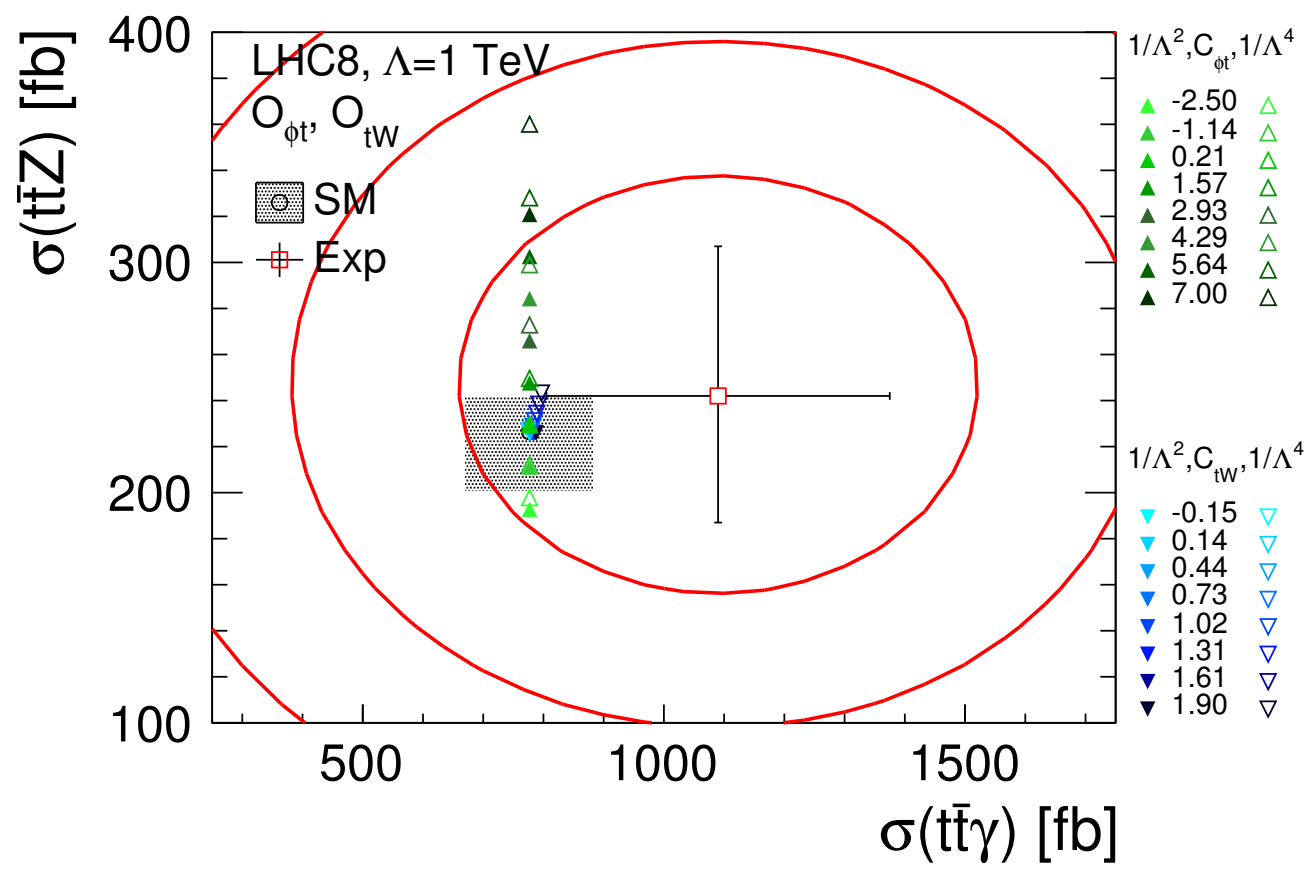

Figure 22. Sensitivity of the $t \bar{t} \gamma$ and $t \bar{t} Z$ processes to the $\mathcal{O}_{\phi t}$ and $\mathcal{O}_{t W}$ operators. Details as in figure 20 . 
from top decay, and it is not expected to be further constrained by $t \bar{t} V$ measurements even with a significant reduction of the experimental uncertainties.

\section{$9 \quad$ Summary and conclusions}

We have presented the NLO QCD predictions in the SMEFT framework for the associated production of a top-quark pair and a neutral gauge boson at the LHC. In addition, we have considered top-pair production in $e^{+} e^{-}$colliders and the top loop-induced process $g g \rightarrow H Z$ at the LHC. These processes are important because they directly probe the neutral gauge-boson couplings to the top quark, which are not well probed by other means. In our approach we have included the full set of dimension-six operators that parameterise these couplings.

We have studied the contribution of each relevant dimension-six operator, in both total cross sections and differential distributions. We have presented full results for $\mathcal{O}\left(\Lambda^{-2}\right)$ contributions, along with the squared contribution of each operator at $\mathcal{O}\left(\Lambda^{-4}\right)$. The latter contribution can be used to estimate uncertainties coming from higher order $\mathcal{O}\left(\Lambda^{-4}\right)$ contributions. Scale uncertainties are provided in all cases, and their reduction at NLO reflects the increased precision of our predictions.

In $t \bar{t} \gamma$ and $t \bar{t} Z$, we find that the operator that contributes the most, given our choice of operator normalisation, is the chromomagnetic one. This observation is particularly important in the context of a global EFT fit, because it means that, when extracting information on operators modifying the top couplings with the weak gauge bosons, uncertainties due to a possible non-vanishing chromomagnetic operator should be carefully accounted for. We also find that, the weak dipole operators give extremely suppressed contributions at $\mathcal{O}\left(1 / \Lambda^{2}\right)$, due to a momentum suppression from the operator structure, and in $t \bar{t} Z$ an additional accidental cancellation between $g g$ and $q \bar{q}$ initial states.

A subset of the operators affects the associated production of the Higgs with a $Z$ in gluon fusion, and we have considered their effects on this process at the LHC. This might provide additional constraints on the operators once $Z H$ production is measured accurately at the LHC. Again, we find that the contribution of $\mathcal{O}_{t G}$ is large, while all the current operators give the same contribution as they affect the axial vector of the $\mathrm{Z}$ in the same way. The weak dipole operators do not contribute due to charge conjugation parity. We have also found that at the ILC, $t \bar{t}$ production is sensitive to weak dipole operators, and could provide information complementary to the LHC.

We have studied the sensitivity of the processes to the various operators in light of the current experimental measurements, as well as the constraints currently placed on the operators from other top measurements and electroweak precision observables. A discussion of the relevant uncertainties coming from missing higher orders in QCD and in the EFT has also been presented. The NLO results provide a solid basis for current and future measurements to be analysed in an EFT approach.

In summary, at NLO in QCD accuracy, deviations from the SM in the top sector can be extracted with improved accuracy and precision, keeping EFT uncertainties under control. As our calculation is based on the MG5_AMC framework, matching with the 
parton shower and top decays with spin correlations can be achieved in an automatic way. Therefore, the corresponding simulations can be directly used in experimental analyses in the future to provide reliable information on possible EFT signals. Furthermore, dedicated investigations of the features of deviations from the SM in these processes can be performed based on our results, with an expected improvement in sensitivity.

\section{Acknowledgments}

We acknowledge illuminating discussions with Christophe Grojean, Alex Pomarol, Francesco Riva on the SMEFT and its range of validity. We would like to thank Raoul Röntsch and Markus Schulze for discussions and helpful checks. C.Z. would like to thank Valentin Hirschi and Hua-Sheng Shao for valuable discussions about gauge anomaly. This work has been performed in the framework of the ERC Grant No. 291377 "LHCTheory" and has been supported in part by the European Union as part of the FP7 Marie Curie Initial Training Network MCnetITN (PITN-GA-2012-315877). C.Z. is supported by the United States Department of Energy under Grant Contracts DE-SC0012704.

\section{A Connection with "anomalous coupling" approach}

In order to compare with other work in the literature, we present here the connection of the Wilson coefficients with the top quark anomalous couplings.

The anomalous coupling approach is followed in $[18,19]$ where the $t \bar{t} Z$ process is used to probe anomalous top couplings. Compared with the anomalous coupling parametrisation of the $\bar{t} t Z$ vertex,

$$
\mathcal{L}_{t t Z}=e \bar{u}\left(p_{t}\right)\left[\gamma^{\mu}\left(C_{1, V}^{Z}+\gamma_{5} C_{1, A}^{Z}\right)+\frac{i \sigma^{\mu \nu} q_{\nu}}{m_{Z}}\left(C_{2, V}^{Z}+i \gamma_{5} C_{2, A}^{Z}\right)\right] v\left(p_{\bar{t}}\right) Z_{\mu}
$$

the relation between anomalous couplings and Wilson coefficients are:

$$
\begin{aligned}
C_{1, V}^{Z} & =\frac{1}{2}\left(C_{\varphi Q}^{(3)}-C_{\varphi Q}^{(1)}-C_{\varphi t}\right) \frac{m_{t}^{2}}{\Lambda^{2} s_{W} c_{W}} \\
C_{1, A}^{Z} & =\frac{1}{2}\left(-C_{\varphi Q}^{(3)}+C_{\varphi Q}^{(1)}-C_{\varphi t}\right) \frac{m_{t}^{2}}{\Lambda^{2} s_{W} c_{W}} \\
C_{2, V}^{Z} & =\left(C_{t W} c_{W}^{2}-C_{t B} s_{W}^{2}\right) \frac{2 m_{t} m_{Z}}{\Lambda^{2} s_{W} c_{W}} \\
C_{2, A}^{Z} & =0
\end{aligned}
$$

Similar relations for the top photon interactions are:

$$
\begin{aligned}
\mathcal{L}_{t t \gamma} & =e \bar{u}\left(p_{t}\right)\left[Q_{t} \gamma^{\mu}+\frac{i \sigma^{\mu \nu} q_{\nu}}{m_{Z}}\left(C_{2, V}^{\gamma}+i \gamma_{5} C_{2, A}^{\gamma}\right)\right] v\left(p_{\bar{t}}\right) A_{\mu} \\
C_{2, V}^{\gamma} & =\left(C_{t W}+C_{t B}\right) \frac{2 m_{t} m_{Z}}{\Lambda^{2}} \\
C_{2, A}^{\gamma} & =0
\end{aligned}
$$

The CP-odd operators are zero simply because we have assumed $C_{t W}$ and $C_{t B}$ are real. 


\section{B Ratios for comparing with measurements}

\section{B.1 ATLAS $-t \bar{t} Z$}

The SM prediction employed by ATLAS for the $t \bar{t} Z$ process [6] also contains contribution of $t \bar{t} \gamma^{*}$ in the channel where the vector boson decays into two charged leptons $\left(t \bar{t} l^{+} l^{-}\right)$. The total prediction, here called $\sigma_{A T L A S}^{S M}(t \bar{t} Z)$, can be written in terms of the $t \bar{t} Z$ and $t \bar{t} l^{+} l^{-}$ processes as follows:

$$
\sigma_{A T L A S}^{S M}(t \bar{t} Z)=\sigma^{S M}\left(t \bar{t} \ell^{+} \ell^{-}, m(\ell \ell)>5 \mathrm{GeV}\right)+\sigma^{S M}(t \bar{t} Z) \times\left[1-B R\left(Z \rightarrow \ell^{+} \ell^{-}\right)\right] .
$$

The $B R\left(Z \rightarrow \ell^{+} \ell^{-}\right)$is taken from MADSPIN [50]. The branching ratio and the NLO cross sections including the absolute scale uncertainties, using our parameter settings, are

$$
\begin{aligned}
\sigma^{S M}\left(t \bar{t} \mu^{+} \mu^{-}, m(\ell \ell)>5 \mathrm{GeV}\right) & =11.63(1)_{-1.38}^{+1.00} \mathrm{fb} \\
\sigma^{S M}\left(t \bar{t} \mu^{+} \mu^{-}, m(\ell \ell)>10 \mathrm{GeV}\right) & =9.83(1)_{-1.13}^{+0.75} \mathrm{fb} \\
\sigma^{S M}(t \bar{t} Z) & =226.5(6)_{-25.3}^{+15.1} \mathrm{fb} \\
B R\left(Z \rightarrow \ell^{+} \ell^{-}\right) & =0.1029 .
\end{aligned}
$$

Applying these results to eq. (B.1), the corresponding prediction when using the same scales, PDF sets and generation procedure as in this paper is:

$$
\sigma_{A T L A S}^{S M}(t \bar{t} Z)=238.1(6)_{-26.8}^{+16.6} \mathrm{fb} .
$$

In order to compare our $t \bar{t} \mu^{+} \mu^{-}$results with the ATLAS measurement we apply to the experimental result the $R_{A T L A S}^{t \bar{t} Z}$, defined as

$$
R_{A T L A S}^{t \bar{t} Z}=\frac{\sigma^{S M}\left(t \bar{t} \mu^{+} \mu^{-}, m(\ell \ell)>10 \mathrm{GeV}\right)}{\sigma_{A T L A S}^{S M}(t \bar{t} Z)}=0.0413(1)_{-0.0001}^{+0.0003} .
$$

The corresponding value for $13 \mathrm{TeV}$ is

$$
R_{A T L A S}^{t \bar{t} Z, 13 \mathrm{TeV}}=0.0408(1)_{-0.0002}^{+0.0003} .
$$

\section{B.2 CMS $-t \bar{t} \gamma$}

The measurement of $t \bar{t} \gamma$ described in ref. [2] should be compared with the $W^{+} b W^{-} \bar{b} \gamma$ SM cross section calculated with $p_{T}(\gamma)>20 \mathrm{GeV}$ and $\Delta R(\gamma, b / \bar{b})>0.1$. Our $t \bar{t} \gamma$ results are with $p_{T}(\gamma)>20 \mathrm{GeV}$, but they do not include photon radiation from the $t, \bar{t}$ decay products $\left(W^{ \pm}, b, \bar{b}\right)$. For this reason the $R_{C M S}^{t \bar{t} \gamma}$ value is applied to the experimental result, defined at $\mathrm{LO}$ as follows

$$
R_{C M S}^{t \bar{t} \gamma}=\frac{\sigma^{S M}\left(t \bar{t} \gamma, p_{T}(\gamma)>20 \mathrm{GeV}\right)}{\sigma^{S M}\left(W^{+} b W^{-} \bar{b} \gamma, p_{T}(\gamma)>20 \mathrm{GeV}, \Delta R(\gamma, b / \bar{b})>0.1\right)}=0.4531(4)_{-0.0011}^{+0.0015} .
$$

The LO cross sections are

$$
\begin{aligned}
\sigma^{S M}\left(t \bar{t} \gamma, p_{T}(\gamma)>20 \mathrm{GeV}\right) & =604.0(3)_{-154.8}^{+234.1} \mathrm{fb} \\
\sigma^{S M}\left(W^{+} b W^{-} \bar{b} \gamma, p_{T}(\gamma)>20 \mathrm{GeV}, \Delta R(\gamma, b / \bar{b})>0.1\right) & =1333.0(9)_{-344.9}^{+520.9} \mathrm{fb} .
\end{aligned}
$$

The corresponding value for $13 \mathrm{TeV}$ is

$$
R_{C M S}^{t \bar{t} \gamma, 13 \mathrm{TeV}}=0.4453(5)_{-0.0003}^{+0.0008} .
$$


Open Access. This article is distributed under the terms of the Creative Commons Attribution License (CC-BY 4.0), which permits any use, distribution and reproduction in any medium, provided the original author(s) and source are credited.

\section{References}

[1] CDF collaboration, T. Aaltonen et al., Evidence for tit $\gamma$ production and measurement of $\sigma_{t \bar{t} \gamma} / \sigma_{t \bar{t}}$, Phys. Rev. D 84 (2011) 031104 [arXiv: 1106.3970] [InSPIRE].

[2] CMS collaboration, Measurement of the inclusive top-quark pair + photon production cross section in the muon + jets channel in pp collisions at $8 \mathrm{TeV}$, CMS-PAS-TOP-13-011, CERN, Geneva Switzerland (2013).

[3] ATLAS collaboration, Observation of top-quark pair production in association with a photon and measurement of the $t \bar{t} \gamma$ production cross section in pp collisions at $\sqrt{s}=7 \mathrm{TeV}$ using the ATLAS detector, Phys. Rev. D 91 (2015) 072007 [arXiv:1502.00586] [InSPIRE].

[4] CMS collaboration, Measurement of top quark-antiquark pair production in association with $a W$ or $Z$ boson in pp collisions at $\sqrt{s}=8$ TeV, Eur. Phys. J. C 74 (2014) 3060 [arXiv: 1406.7830] [INSPIRE].

[5] CMS collaboration, Observation of top quark pairs produced in association with a vector boson in pp collisions at $\sqrt{s}=8 \mathrm{TeV}$, JHEP 01 (2016) 096 [arXiv:1510.01131] [INSPIRE].

[6] ATLAS collaboration, Measurement of the $t \bar{t} W$ and $t \bar{t} Z$ production cross sections in $p p$ collisions at $\sqrt{s}=8 \mathrm{TeV}$ with the ATLAS detector, JHEP 11 (2015) 172 [arXiv: 1509.05276] [INSPIRE].

[7] S. Weinberg, Phenomenological Lagrangians, Physica A 96 (1979) 327 [InSPIRE].

[8] W. Buchmüller and D. Wyler, Effective Lagrangian analysis of new interactions and flavor conservation, Nucl. Phys. B 268 (1986) 621 [INSPIRE].

[9] C.N. Leung, S.T. Love and S. Rao, Low-energy manifestations of a new interaction scale: operator analysis, Z. Phys. C 31 (1986) 433 [INSPIRE].

[10] G. Durieux, F. Maltoni and C. Zhang, Global approach to top-quark flavor-changing interactions, Phys. Rev. D 91 (2015) 074017 [arXiv:1412.7166] [InSPIRE].

[11] A. Buckley et al., Global fit of top quark effective theory to data, Phys. Rev. D 92 (2015) 091501 [arXiv: 1506.08845] [INSPIRE].

[12] A. Buckley et al., Constraining top quark effective theory in the LHC Run II era, JHEP 04 (2016) 015 [arXiv: 1512.03360] [INSPIRE].

[13] C. Zhang and F. Maltoni, Top-quark decay into Higgs boson and a light quark at next-to-leading order in QCD, Phys. Rev. D 88 (2013) 054005 [arXiv:1305.7386] [InSPIRE].

[14] C. Zhang, Effective field theory approach to top-quark decay at next-to-leading order in QCD, Phys. Rev. D 90 (2014) 014008 [arXiv: 1404.1264] [InSPIRE].

[15] C. Degrande, F. Maltoni, J. Wang and C. Zhang, Automatic computations at next-to-leading order in QCD for top-quark flavor-changing neutral processes, Phys. Rev. D 91 (2015) 034024 [arXiv: 1412 . 5594] [INSPIRE].

[16] D. Buarque Franzosi and C. Zhang, Probing the top-quark chromomagnetic dipole moment at next-to-leading order in QCD, Phys. Rev. D 91 (2015) 114010 [arXiv:1503.08841] [INSPIRE]. 
[17] C. Zhang, Single top production at next-to-leading order in the standard model effective field theory, Phys. Rev. Lett. 116 (2016) 162002 [arXiv:1601.06163] [INSPIRE].

[18] R. Röntsch and M. Schulze, Constraining couplings of top quarks to the $Z$ boson in $t \bar{t}+Z$ production at the LHC, JHEP 07 (2014) 091 [Erratum ibid. 09 (2015) 132] [arXiv: 1404.1005] [INSPIRE].

[19] R. Röntsch and M. Schulze, Probing top-Z dipole moments at the LHC and ILC, JHEP 08 (2015) 044 [arXiv: 1501.05939] [INSPIRE].

[20] J. Alwall et al., The automated computation of tree-level and next-to-leading order differential cross sections and their matching to parton shower simulations, JHEP 07 (2014) 079 [arXiv: 1405.0301] [INSPIRE].

[21] C. Zhang, Automating predictions for standard model effective field theory in MadGraph5_aMC@NLO, arXiv:1601.03994 [INSPIRE].

[22] J.A. Aguilar-Saavedra, A minimal set of top anomalous couplings, Nucl. Phys. B 812 (2009) 181 [arXiv:0811.3842] [INSPIRE].

[23] B. Grzadkowski, M. Iskrzynski, M. Misiak and J. Rosiek, Dimension-six terms in the standard model Lagrangian, JHEP 10 (2010) 085 [arXiv: 1008.4884] [INSPIRE].

[24] C. Zhang and S. Willenbrock, Effective-field-theory approach to top-quark production and decay, Phys. Rev. D 83 (2011) 034006 [arXiv: 1008.3869] [INSPIRE].

[25] C. Degrande, J.-M. Gerard, C. Grojean, F. Maltoni and G. Servant, Non-resonant new physics in top pair production at hadron colliders, JHEP 03 (2011) 125 [arXiv:1010.6304] [INSPIRE].

[26] J.A. Aguilar-Saavedra, B. Fuks and M.L. Mangano, Pinning down top dipole moments with ultra-boosted tops, Phys. Rev. D 91 (2015) 094021 [arXiv:1412.6654] [InSPIRE].

[27] A. Tonero and R. Rosenfeld, Dipole-induced anomalous top quark couplings at the LHC, Phys. Rev. D 90 (2014) 017701 [arXiv:1404.2581] [INSPIRE].

[28] C. Zhang, N. Greiner and S. Willenbrock, Constraints on non-standard top quark couplings, Phys. Rev. D 86 (2012) 014024 [arXiv: 1201.6670] [InSPIRE].

[29] N. Greiner, S. Willenbrock and C. Zhang, Effective field theory for nonstandard top quark couplings, Phys. Lett. B 704 (2011) 218 [arXiv:1104.3122] [INSPIRE].

[30] J. de Blas, M. Chala and J. Santiago, Renormalization group constraints on new top interactions from electroweak precision data, JHEP 09 (2015) 189 [arXiv:1507.00757] [INSPIRE].

[31] A. Alloul, N.D. Christensen, C. Degrande, C. Duhr and B. Fuks, FeynRules $2.0-a$ complete toolbox for tree-level phenomenology, Comput. Phys. Commun. 185 (2014) 2250 [arXiv: 1310.1921] [INSPIRE].

[32] C. Degrande, C. Duhr, B. Fuks, D. Grellscheid, O. Mattelaer and T. Reiter, UFO - the Universal FeynRules Output, Comput. Phys. Commun. 183 (2012) 1201 [arXiv:1108.2040] [INSPIRE].

[33] C. Degrande, Automatic evaluation of $U V$ and $R_{2}$ terms for beyond the standard model Lagrangians: a proof-of-principle, Comput. Phys. Commun. 197 (2015) 239 [arXiv:1406.3030] [INSPIRE]. 
[34] V. Hirschi, R. Frederix, S. Frixione, M.V. Garzelli, F. Maltoni and R. Pittau, Automation of one-loop QCD corrections, JHEP 05 (2011) 044 [arXiv: 1103.0621] [INSPIRE].

[35] R. Frederix, S. Frixione, F. Maltoni and T. Stelzer, Automation of next-to-leading order computations in QCD: the FKS subtraction, JHEP 10 (2009) 003 [arXiv:0908.4272] [INSPIRE].

[36] V. Hirschi and O. Mattelaer, Automated event generation for loop-induced processes, JHEP 10 (2015) 146 [arXiv:1507.00020] [INSPIRE].

[37] T. Sjöstrand et al., An introduction to PYTHIA 8.2, Comput. Phys. Commun. 191 (2015) 159 [arXiv:1410.3012] [INSPIRE].

[38] M. Bahr et al., HERWIG++ physics and manual, Eur. Phys. J. C 58 (2008) 639 [arXiv:0803.0883] [INSPIRE].

[39] S. Frixione and B.R. Webber, Matching NLO QCD computations and parton shower simulations, JHEP 06 (2002) 029 [hep-ph/0204244] [INSPIRE].

[40] E.E. Jenkins, A.V. Manohar and M. Trott, Renormalization group evolution of the standard model dimension six operators I: formalism and $\lambda$ dependence, JHEP 10 (2013) 087 [arXiv: 1308.2627] [INSPIRE].

[41] E.E. Jenkins, A.V. Manohar and M. Trott, Renormalization group evolution of the standard model dimension six operators II: Yukawa dependence, JHEP 01 (2014) 035 [arXiv: 1310.4838] [INSPIRE].

[42] R. Alonso, E.E. Jenkins, A.V. Manohar and M. Trott, Renormalization group evolution of the standard model dimension six operators III: gauge coupling dependence and phenomenology, JHEP 04 (2014) 159 [arXiv:1312.2014] [INSPIRE].

[43] J. Preskill, Gauge anomalies in an effective field theory, Annals Phys. 210 (1991) 323 [INSPIRE].

[44] O. Domenech, A. Pomarol and J. Serra, Probing the SM with dijets at the LHC, Phys. Rev. D 85 (2012) 074030 [arXiv:1201.6510] [INSPIRE].

[45] A. Biekötter, A. Knochel, M. Krämer, D. Liu and F. Riva, Vices and virtues of Higgs effective field theories at large energy, Phys. Rev. D 91 (2015) 055029 [arXiv:1406.7320] [InSPIRE].

[46] A.D. Martin, W.J. Stirling, R.S. Thorne and G. Watt, Parton distributions for the LHC, Eur. Phys. J. C 63 (2009) 189 [arXiv:0901.0002] [INSPIRE].

[47] F. Maltoni, D. Pagani and I. Tsinikos, Associated production of a top-quark pair with vector bosons at NLO in QCD: impact on $t \bar{t} H$ searches at the LHC, JHEP 02 (2016) 113 [arXiv: 1507.05640] [INSPIRE].

[48] S. Frixione, Isolated photons in perturbative QCD, Phys. Lett. B 429 (1998) 369 [hep-ph/9801442] [INSPIRE].

[49] S. Frixione, V. Hirschi, D. Pagani, H.S. Shao and M. Zaro, Electroweak and QCD corrections to top-pair hadroproduction in association with heavy bosons, JHEP 06 (2015) 184 [arXiv: 1504.03446] [INSPIRE].

[50] P. Artoisenet, R. Frederix, O. Mattelaer and R. Rietkerk, Automatic spin-entangled decays of heavy resonances in Monte Carlo simulations, JHEP 03 (2013) 015 [arXiv:1212.3460] [INSPIRE]. 
[51] J.A. Dror, M. Farina, E. Salvioni and J. Serra, Strong tW scattering at the LHC, JHEP 01 (2016) 071 [arXiv: 1511.03674] [InSPIRE].

[52] B. Hespel, F. Maltoni and E. Vryonidou, Higgs and $Z$ boson associated production via gluon fusion in the SM and the $2 H D M$, JHEP 06 (2015) 065 [arXiv:1503.01656] [INSPIRE].

[53] L. Berthier and M. Trott, Consistent constraints on the standard model effective field theory, JHEP 02 (2016) 069 [arXiv: 1508.05060] [INSPIRE].

[54] CMS collaboration, Combination of ATLAS and CMS top quark pair cross section measurements in the emu final state using proton-proton collisions at $8 \mathrm{TeV}$, CMS-PAS-TOP-14-016, CERN, Geneva Switzerland (2014).

[55] M. Czakon, P. Fiedler and A. Mitov, Total top-quark pair-production cross section at hadron colliders through $O\left(\alpha_{S}^{4}\right)$, Phys. Rev. Lett. 110 (2013) 252004 [arXiv: 1303.6254] [INSPIRE]. 